\title{
From local interactions to population dynamics in site-based models of ecology
}

\author{
A. Johansson ${ }^{\mathrm{a}}$ and D.J.T. Sumpter ${ }^{\mathrm{a}, \mathrm{b}, *}$ \\ ${ }^{a}$ Mathematics Department, Umeå University, SE-901 87 Umeå, Sweden

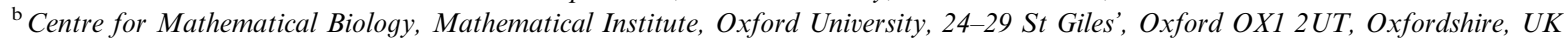

Received 21 May 2003

\begin{abstract}
A central problem in ecology is relating the interactions of individuals - described in terms of competition, predation, interference, etc. - to the dynamics of the populations of these individuals - in terms of change in numbers of individuals over time. Here, we address this problem for a class of site-based ecological models, where local interactions between individuals take place at a finite number of discrete resource sites over non-overlapping generations and, between generations, individuals move randomly between sites over the entire system. Such site-based models have previously been applied to a wide range of ecological systems: from those involving contest or scramble competition for resources to host-parasite interactions and meta-populations. We show how the population dynamics of site-based models can be accurately approximated by and understood through deterministic and stochastic difference equations. Conversely, we use the inverse of this approximation to show what implicit assumptions are made about individual interactions by modelling of population dynamics in terms of difference equations. To this end, we prove a useful and general theorem: that any model in our class of site-based models has a corresponding stochastic difference equation population model, by which it can be approximated. This theorem allows us to calculate long-term population dynamics, evolutionary stable strategies and, by extending our theory to account for large deviations, extinction probabilities for a wide range of site-based systems. Our methodology is then illustrated to various examples of between species competition, predator-prey interactions and co-operation.
\end{abstract}

(C) 2003 Elsevier Inc. All rights reserved.

Keywords: Site-based; Individual-based; Stochastic difference equations; Population dynamics

\section{Introduction}

In the past 10 years, a shift has occurred in theoretical ecology away from 'top-down', phenomenological models - aimed at capturing general properties of an ecological system-toward 'bottom-up', individualbased models - built on the behavioural algorithms employed by interacting and reproducing individuals in a spatial environment (Diekmann et al., 2000). Although individual-based thinking has undoubtedly been facilitated by increased computer power and accessibility of the techniques involved, the main reason for the shift is that the individual-based approach accounts much more fully for the intricacies and details

${ }^{*}$ Corresponding author. Centre for Mathematical Biology, Mathematical Institute, Oxford University, 24-29 St Giles', Oxford OX1 2UT, Oxfordshire, UK. Fax: +44-1865-270515.

E-mail address: sumpter@maths.ox.ac.uk (D.J.T. Sumpter). considered key in determining how an ecological system functions. In accounting for these details, the number of predictions made about the behaviour and evolution of ecological systems has rapidly increased (Ermentrout and Edelstein-Keshet, 1992; Nowak and May, 1992; Boerlijst et al., 1993; Bolker and Grenfell, 1995; Pacala and Tilman, 1996; Iwasa et al., 1998; Keeling et al., 2000). More recently, the individual-based approach has been brought to bear on making specific predictions about systems - in particular to epidemiology and micro-bacterial ecology - where data can be collected at fine enough individual and spatial resolution to validate models (Keeling and Gilligan, 2000; Keeling et al., 2001; Kerr et al., 2002). As the theoretical basis of individual-based modelling has matured it has become a very popular paradigm for understanding and predicting the behaviour of ecological systems (see the text books of, for example, DeAngelis and Gross, 1992; Czaran, 1998). 
An early criticism of the individual-based approach was that models do not have the analytic tractability of top-down dynamical system models. This led to the development of numerous methods, ranging from simple mean-field approximations to moment closure techniques, for simplifying and analysing individualbased models (Durrett and Levin, 1994a; Rand, 1999; Keeling et al., 2002). In the simplest of individual-based models-where although there are discrete, separate interaction sites there is no spatial structure and interactions are simple births and deaths - a relationship can be shown between the individual-based model and an appropriately chosen deterministic dynamical system. For example, Levins $(1969,1970)$ meta-population model is at first described in terms of $n$ individual patches of which a proportion $p$ are occupied. Each individual patch is independent, going extinct stochastically at a rate $e$ and produces offspring at rate $c$. These offspring occupy an empty patch with probability $1-p$. As $n \rightarrow \infty$, the dynamics of the proportion of occupied patches is

$\frac{d p}{d t}=c p(1-p)-e p$

which is identical to the 'top-down' logistic model for continuous population growth. This approximation holds even when $n$ is reasonably small, i.e. over 50 , and has been shown to apply, at least in terms of its predicted relationship between number of occupied patches and rate of extinction, even when more detailed within-patch population dynamics are taken into account (Keeling, 2002). Levins' meta-population model provides one, of possibly many, 'first principles' justifications of the continuous time logistic equation in terms of interactions between individuals. Instead of stating a particular carrying capacity, $K$, to which the population grows - as is the case when the logistic equation is used as a 'top-down' phenomenological model - Levins' model gives the carrying capacity, $K=$ $1-e / c$, in terms of local births and deaths.

Through a similar mode of reasoning, Royama (1992) considered an individual-based model where reproducing individuals are distributed on discrete generations completely at random over a uniform resource environment. Each of these individuals produce offspring as a function of the number of the neighbours, $a$, within some radius, $r$, such that the number of offspring $B=$ $B_{0} c^{a}$, where $0 \leqslant c<1$ and $B_{0}>0$ are constants. Because the individuals are randomly distributed, $a$ follows a Poisson distribution. Using this observation, Royama showed that, when the global population density is $P_{t}$, the total number of offspring produced by the population to go on to the next generation is

$P_{t+1}=B_{0} P_{t} e^{-r(1-c) P_{t}}$

which is the exponential logistic equation or Ricker map. Again the parameters in the individual-based model are linked to a 'top-down' model. In this case, the carrying capacity for the population is $\ln \left(B_{0}\right) /(r(1-c))$. Such a 'first principles' justification of the Ricker map can also be argued in terms of local competition between individuals distributed amongst a finite number of discrete resource sites (Sumpter and Broomhead (2001) and see later in this article). The method employed by Royama was also employed independently and greatly extended in a series of papers by Pacala on local plant interactions (Pacala and Silander, 1985), who added complexities such as more than one plant species (Pacala, 1986a), different seed dormancy times (Pacala, 1986b) and age and size structure. Each of these individual-based models had its own approximating dynamical system of equations.

In the above examples, a relationship has been established between an individual-based model and a dynamical system. In both cases, the approximation which established the relationship relied on the lack of spatial structure of the system: although at some stage, individuals interacted locally there was also a stage at which they moved at random across the entire system. In Royama's (1992) model, competition occurs between a small number of individuals within radius $r$ of each other, but migrating offspring chose a position at random over a uniform resource environment. Deriving such approximations is not trivial, in particular when local interactions are complicated and non-linear. The discreteness of individuals and small finite populations at the stage of local interactions produces fluctuations that cause deviations from models where all interactions are described simply in terms of whole populations (Durrett and Levin, 1994b; Diekmann and Law, 2000). Furthermore, individual-based models with finite populations are subject to stochastic effects, such as extinction or flipping between alternate stable states, which population approximations do not account for.

The aim of this article is to give a complete and general treatment of individual-based models where, although there is no spatial structure in the population, local interactions between individuals determine the population dynamics. Specifically, we consider a class of individual-based models, which, following Czaran (1998), we call well-mixed site-based models: where on each discrete generation, individuals of one or more different species or phenotype are distributed uniformly at random across some finite number of discrete resource sites. The individuals then interact locallycompete, co-operate, prey upon each other, reproduce, etc. - at these sites. A specific model is defined by its interaction rules. These interaction rules determine which individuals survive and reproduce and which die. The offspring produced through the interaction pass to the next generation and are again distributed at random amongst resource sites, and the process repeats. The class of site-based models we propose are general in 
the sense that any type of interaction, stochastic or deterministic, between individuals is permitted.

A large class of systems in ecology fall into a category that can be modelled using well-mixed site-based models. There are many examples of organisms, often parasitoids, competing for multiple discrete resource sites or hosts. Tachinid fly larvae developing inside butterflies (Iwao and Ohsaki, 1996), Varroa mites feeding on honey bee brood (Rath, 1999), bruchid beetles on beans (Toquenaga, 1993), blow-flies on carcasses (Prinkkila and Hanski, 1995) and caterpillars in ants nests (Thomas et al., 1993) all involve individual organisms occupying a resource site then competing with other individuals which have also occupied it. In other systems, competition is complicated by the existence of two or more species each with a different form of interaction. For example, different species of bruchid beetles show scramble-where a resource is partitioned dependent on the number of competing individuals - and contest - where a single individual takes the entire resource - competition (Toquenaga and Fujii, 1990a; Takano et al., 2001). The outcome in terms of offspring at each resource site depends on the number of each species occupying each site (Toquenaga and Fujii, 1990b, 1991). In other cases, the competing species is itself parasitised. For example, Tuda (1998) studied another bruchid species, the cow-pea weevil, which are themselves host to braconid wasps. This system is thought to be subject to evolution in terms of the types of interactions exhibited by the host weevils on the cow-pea resources, as well as effected by the parasitic actions of the wasps (Tuda and Iwasa, 1998). For the above examples, and for many more, the relationship between number of individuals per resource site and the survivorship and fecundity of individuals produced at a site has been, or could be, established empirically.

Despite the range and complexity of interaction rules which occur in nature, it is possible to prove general and useful results about systems where interactions take place at discrete sites. In this paper, we prove a number of results which are directly applicable to any natural system that can be described through site-based interactions. We show that any site-based model with $s$ interacting species can be accurately approximated by a unique set of $s$ coupled stochastic difference equations. Furthermore, the variance in the approximation, and thus a stochastic term for these difference equations, can be calculated from the interaction rules and the number of sites. In cases when population extinction probabilities are required we show how these can be calculated, as can the probability that a population shifts between two alternative stable regimes.

Our approach links together, in a single coherent framework, two approaches to modelling population dynamics of animals with non-overlapping generations: site-based modelling of individual interactions and difference equations with a stochastic term. As the above examples of meta-populations and Royama's derivation of the Ricker map illustrate, our approach is not completely new. Our aim is rather to give a thorough and general treatment of well-mixed site-based models, focusing on topics such as: large system size approximations by difference equations; first principles derivations of difference equation models; estimating variance in the population density in particular when the number of resource sites is small; calculating extinction probabilities for individual interaction models and structural stability of site-based models. In the next section, we outline the basic theoretical ideas, which are then illustrated in further sections by particular examples.

\section{Site-based models}

The term individual-based models is used to describe a wide range of approaches, from cellular automata (Ermentrout and Edelstein-Keshet, 1992), interacting particle systems (Durrett and Levin, 1994a) to structured meta-population models (Hanski, 1999). As we stated in the introduction, we are concerned here with a particular class of individual-based models where interactions between individuals happen at a finite number of resource sites on discrete generations. We use the term well-mixed site-based models to refer to this class of models, because between generations there is mixing of individuals across the entire system. It is important to note then, that the results discussed here are not directly applicable to all individual-based models, in particular not to those where individuals disperse only locally. However, many individual-based models which are not explicitly spatial or in which individuals disperse over large distances relative to the size of the entire system will have an analogue as a wellmixed site-based model.

We now define mathematically the type of models we will consider and state the results we have proved about these models: the examples in the next section will further illuminate the usefulness of these results in specific cases. On first reading it may be helpful to skip straight to these examples, and in particular Fig. 1 and the accompanying description of contest competition, before reading the more general model definition given here.

\subsection{Model definition}

Consider a fixed number $n$ of resource sites and $s$ species, which correspond to different animal species or different phenotypes, and which will interact at the sites. 


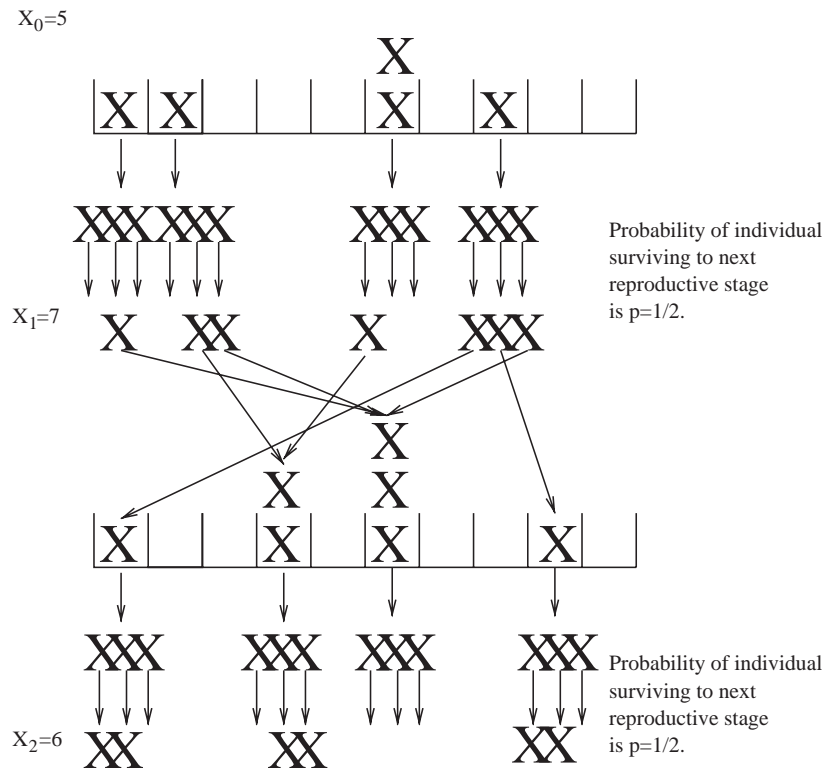

Fig. 1. Diagrammatic representation of a site-based contest model. On generation $t$ the individual animals (each represented by an ' $\mathrm{X}$ ') choose and enter a site (represented by boxes) at random. Only one animal per site may successfully reproduce, producing $b=3$ offspring. Before the next reproductive stage, each of the newly produced animals has probability $p=1 / 2$ of dying. The surviving animals become the $t+1$ generation, and again choose reproductive sites.

On generation $t$ the total community population across all sites of species $i$ is denoted as $M_{i, t}$. The $s$-dimensional vector

$\mathbf{M}_{t}=\left(M_{1, t}, \ldots, M_{s, t}\right) \in \mathbb{Z}^{s}$

thus denotes the state of the total population at time $t$.

The dynamics of $\mathbf{M}_{t}$ are determined by the distribution of the populations amongst resource sites. The $\mathbf{M}_{t}$ individuals are first distributed uniformly at random between the $n$ sites, giving $\mathrm{C}=\left(\mathrm{C}_{1}, \ldots, \mathrm{C}_{n}\right)$, where $\mathrm{C}_{i}=\left(C_{i, 1}, \ldots, C_{i, s}\right)^{\mathrm{T}}$ describes the content of site $i$. The content of each site is then modified according to an interaction. For specific models, the interaction is defined according to how the individuals of different species at site $i$ might breed, compete or prey on each other, etc. The interactions at each site are independent of those at all other sites and are determined by an interaction function, $\phi: \mathbb{Z}^{s} \times \mathbb{Z}^{r} \rightarrow \mathbb{Z}^{s}$. The interaction function acts on the site content along with a vector of $r$ mutually independent, but identically distributed for each site, random variables to give a new site population, $\phi\left(\mathrm{C}_{i}, \xi_{i}\right)$. The purpose or $\xi_{i}$ is to allow between-site variability, giving a stochastic outcome for each site independent of other sites. For example, we may want to specify that, for the same fixed value of $\mathbf{k} \in \mathbb{Z}^{s}, \phi\left(\mathbf{k}, \xi_{i}\right)$ gives two or more different outcomes with the probability of each outcome dependent upon one of the values in $\xi_{i}$.
After interaction, the population at site $i$ is $\phi\left(\mathrm{C}_{i}, \xi_{i}\right)$ and

$\mathbf{M}_{t+1}=\sum_{i=1}^{n} \phi\left(\mathrm{C}_{i}, \xi_{i}\right)$

gives the total population on the next generation. As the state space of the population process we take the vector $\mathbf{X}_{t}:=\mathbf{M}_{t} / n \in \mathbb{R}^{s}$ giving the population densities relative to the number of sites. The population density at time $t+1$ is thus

$\mathbf{X}_{t+1}=\frac{1}{n} \sum_{i=1}^{n} \phi\left(\mathrm{C}_{i}, \xi_{i}\right)$

We now present some results concerning the stochastic dynamical systems defined by Eqs. (1) and (2).

\subsection{Density approximation}

When $n$ is large, Eq. (2) can be approximated by a deterministic dynamical system. The density approximation is based on the expected population of $\mathbf{X}_{t+1}$ which is determined by the following proposition.

Proposition 1. As $n \rightarrow \infty$ if the population density is $\mathbf{X}_{t}=$ $\mathbf{x}=\mathbf{M}_{t} / n$ at time $t$, on generation $t+1$ the expected population density is

$E\left[\mathbf{X}_{t+1} \mid \mathbf{X}_{t}=\mathbf{x}\right]:=\Phi(\mathbf{x})=e^{-\mathbf{x}} \sum_{\mathbf{k} \in \mathbb{Z}^{s}} \frac{\mathbf{x}^{\mathbf{k}}}{\mathbf{k} !} E\left[\phi\left(\mathbf{k}, \xi_{i}\right)\right]$,

where we use the notational convention where, for example, $\mathbf{k} !=k_{1} ! k_{2} ! \ldots k_{s} !$ and $\mathbf{x}^{\mathbf{k}}=x_{1}^{k_{1}} x_{2}^{k_{2}} \ldots x_{s}^{k_{s}}$. The proof of Proposition 1 is based on the fact that the distribution of the population at each site, $C_{i}$, is 'similar' to a that of a vector of Poisson random variables, $U_{i}$ each with mean $n \mathbf{x}$. In particular, as $n$ becomes large the sum of the expected $U_{i}$ 's and the sum of $\mathrm{C}_{i}$ 's have the same mean. A full proof is given in the appendix.

Proposition 1 can be used to define a deterministic dynamical system: initial condition $\mathbf{x}_{0}=\mathbf{X}_{0}$ and, for $t \geqslant 0$,

$\mathbf{x}_{t+1}=\Phi\left(\mathbf{x}_{t}\right)$

which we call the density approximation of Eq. (2) for the population density. This density approximation will prove the most common focus of our study of population dynamics. However, an approximation of the absolute number of individuals of each species, from Eq. (1), is often useful. The dynamical system

$\mathbf{m}_{t+1}=n \Phi\left(\mathbf{m}_{t} / n\right)$

gives such an approximation.

\subsection{Normal approximation}

Site-based models are inherently stochastic while the density approximation gives an approximation only of 
mean behaviour. It is thus important to establish how large the fluctuations are around this approximation. The following theorem gives us the variance of these fluctuations:

Theorem 2. As $n \rightarrow \infty$,

$\frac{1}{\sqrt{n}}\left(\sum_{i=1}^{n} \phi\left(\mathrm{C}_{i}, \xi_{i}\right)-n \Phi(\mathbf{x})\right)$

converges in distribution to a normal distribution with mean $\mathbf{0}$ and variance

$v(\mathbf{x})=\operatorname{Var}[\phi]-\frac{\partial \Phi}{\partial \mathbf{x}}(\operatorname{diag} \mathbf{x}) \frac{\partial \Phi^{\mathrm{T}}}{\partial \mathbf{x}}$,

where the variance $\operatorname{Var}[\phi]$ is defined as the $s \times s$ matrix

$e^{-\mathbf{x}} \sum_{\mathbf{k} \in \mathbb{Z}^{s}} \frac{\mathbf{x}^{\mathbf{k}}}{\mathbf{k} !} E\left[\phi\left(\mathbf{k}, \xi_{i}\right) \phi\left(\mathbf{k}, \xi_{i}\right)^{\mathrm{T}}\right]-\Phi(\mathbf{x}) \Phi(\mathbf{x})^{\mathrm{T}}$.

$\Phi(\mathbf{x})$ is given by Eq. (3) above; $\frac{\partial \Phi}{\partial \mathbf{x}}$ is the Jacobian matrix of partial derivatives of $\Phi(\mathbf{x})$; and diag $\mathbf{x}$ is the diagonal matrix having $\mathbf{x}=\left(x^{1}, \ldots, x^{s}\right)$ along the diagonal.

For a single species, $s=1$,

$v(x)=e^{-x} \sum_{k=1}^{\infty} \frac{x^{k}}{k !} E\left[\phi\left(k, \xi_{i}\right)^{2}\right]-\Phi(x)^{2}-x\left(\frac{d \Phi}{d x}\right)^{2}$.

The proof of Theorem 2 is based on the fact that the distribution of the population at each site, $C_{i}$, is identical to that of a vector of Poisson random variables, $\mathrm{U}_{i}$, each with mean $\mathbf{x}$, conditioned on $\sum_{i=1}^{n} \cup_{i}=n \mathbf{x}$. The term $\operatorname{Var}[\phi]$ is thus the variance for the $U_{i}$ 's while the adjustment involving the Jacobian of $\Phi$ accounts for the conditioning that all the $\mathrm{U}_{i}$ sum to $n \mathbf{x}$. A full proof of Theorem 2 is given in the appendix.

We can use Theorem 2 to define a stochastic dynamical system which approximates $\mathbf{X}_{t}$. Since, by definition in Eq. (2), $\mathbf{X}_{t+1}=\frac{1}{n} \sum_{i=1}^{n} \phi\left(\mathrm{C}_{i}, \xi_{i}\right)$, for sufficiently large $n$, the stochastic dynamical system

$\mathbf{x}_{t+1}=\Phi\left(\mathbf{x}_{t}\right)+\frac{1}{\sqrt{n}} \varepsilon_{t}\left(\mathbf{x}_{t}\right)$,

where $\varepsilon_{t}\left(\mathbf{x}_{t}\right) \sim N\left(0, v\left(\mathbf{x}_{t}\right)\right)$ (multivariate normally distributed) will give a satisfactory description of $\mathbf{X}_{t+1}$. Furthermore, since the standard deviation of $\mathbf{X}_{t+1}$ is proportional to $1 / \sqrt{n}$, for even larger $n$, Eq. (4) will give an accurate description of our original site-based model. In other words, as the number of sites increases, fluctuations in the density decrease as $1 / \sqrt{n}$. It should be noted that $n$ does not have to be very large for our approximation to apply. Indeed, in many of the examples we discuss in the next section $n=100$ or 200 and the error in the density approximation of the standard deviation is less than $1 / n$.
A useful corollary (due to D.S. Broomhead, personal communication) gives the stationary distribution of $\mathbf{x}_{t}$ in Eq. (9).

Corollary 3. Given a stable fixed point $\mathbf{x}_{*}$ of the map $\Phi\left(\mathbf{X}_{t}\right)$, if $\mathbf{X}_{0}=\mathbf{x}_{*}$ then as $t \rightarrow \infty$ (but conditioned on no 'improbable excursions' to other stable fixed points), $\mathbf{X}_{t}$ is normally distributed with mean $\mathbf{x}_{*}$ and variance approximately equal to $w\left(\mathbf{x}_{*}\right) / n$, where

$w\left(\mathbf{x}_{*}\right):=\sum_{\tau=0}^{\infty} \mathbf{J}\left(\mathbf{x}_{*}\right)^{\tau} v\left(\mathbf{x}_{*}\right) \mathbf{J}\left(\mathbf{x}_{*}\right)^{\tau^{\mathrm{T}}}$

and $\mathbf{J}\left(\mathbf{x}_{*}\right)=\left.\frac{\partial \Phi}{\partial \mathbf{x}}\right|_{\mathbf{x}=\mathbf{x}_{*}}$ is the Jacobian $\frac{\partial \Phi}{\partial \mathbf{x}}$ evaluated at $\mathbf{x}_{*}$.

For a single species,

$w\left(x_{*}\right)=v\left(x_{*}\right) \sum_{\tau=0}^{\infty}\left(\left.\frac{d \Phi}{d x}\right|_{x=x_{*}}\right)^{2 \tau}=\frac{v\left(x_{*}\right)}{1-\left(\left.\frac{d \Phi}{d x}\right|_{x=x_{*}}\right)^{2}}$.

The distribution of $\mathbf{X}_{t}$ close to $\mathbf{x}_{*}$ is thus approximated by a normal distribution with mean $\mathbf{x}_{*}$ and variance given by Eq. (10). This variance result may be easily extended to find the distribution of $\mathbf{X}_{t}$ when the corresponding $\Phi$ gives periodic orbits. The approximation of $\mathbf{X}_{t}$ is always conditional on no improbable excursions'. We now discuss the probability of such excursions taking place.

\subsection{Large deviations}

Although the normal approximation of a site-based model will prove useful for a wide range of applications, it is not sufficient for calculating how long it will take a population to become extinct. Indeed, such extinctions will occur exactly when the population deviates from 'normal behaviour'. Furthermore, most biological realistic interaction functions will give an absorbing state, $\mathbf{x}=\mathbf{0}$, corresponding to extinction. This absorbing state will usually be the only truly invariant distribution of a sitebased model, with the fixed points of $\Phi$ actually being quasi-stable states around which the population density fluctuates, with deviations of order $1 / \sqrt{n}$, for long periods. A common question of interest is then, how long a population will make these normal fluctuations around the fixed point before, through one or more large jumps, either moving to another stable fixed point or becoming extinct.

The theory of large deviations may be used to estimate the probability of such excursions through large jumps (Dembo and Zeitouni, 1992). For most reasonable interaction functions, it is possible to show that large deviations away from a stable fixed point will occur at a exponentially small frequency, i.e. of the order $O(a)^{n}$, for some $a<1$. To this end, we will define a two-point rate function $I(\mathbf{y}, \mathbf{x})$

$I(\mathbf{y}, \mathbf{x})=\min _{\beta}\{\Lambda(\beta, \mathbf{x})-\langle\beta, \mathbf{y}\rangle\}$ 
where the logarithmic moment generating function $\Lambda$ : $\mathbb{R}^{s} \times \mathbb{R}^{s} \rightarrow \mathbb{R}$ is defined by

$\Lambda(\beta, \mathbf{x})=\log \left(e^{-\mathbf{x}} \sum_{\mathbf{k} \in \mathbb{Z}^{s}} \frac{\mathbf{x}^{\mathbf{k}}}{\mathbf{k} !} \exp \left(\left\langle\beta, E\left[\phi\left(\mathbf{k}, \xi_{i}\right)\right]\right\rangle\right)\right)$.

Note that $\langle\beta, \mathbf{y}\rangle$ is the inner product, $\beta_{1} y_{1}+\cdots+\beta_{s} y_{s}$, of $\beta$ and $\mathbf{y}$. For a single species,

$I(y, x)=\min _{\beta}\left\{\log \left(\sum_{k=0}^{\infty} \frac{x^{k}}{k !} e^{\beta E\left[\phi\left(k, \xi_{i}\right)\right]}\right)-x-\beta y\right\}$

is the rate function. The rate function can sometimes be found by finding the minimum stationary point, $\beta=$ $\beta_{*}(\mathbf{x}, \mathbf{y})$, of

$\frac{\partial \Lambda}{\partial \beta}=\mathbf{y}$

to give $I(\mathbf{y}, \mathbf{x})=\Lambda\left(\beta_{*}(\mathbf{x}, \mathbf{y}), \mathbf{x}\right)-\left\langle\beta_{*}(\mathbf{x}, \mathbf{y}), \mathbf{y}\right\rangle$.

We now state the following large deviation theorem. It says that the rate function, $I(\mathbf{y}, \mathbf{x})$, can be used to estimate an upper bound for the probability of a transition from one part of the population state space to another, and is a direct application of Cramér's theorem,

Theorem 4. As $n \rightarrow \infty$, if $\mathbf{X}_{t} \notin K$ is the number of individuals at time $t$, then

$\operatorname{Pr}\left\{\mathbf{X}_{t+1} \in K \mid \mathbf{X}_{t} \notin K\right\} \leqslant \exp \left(-n\left(I_{K}+o(1)\right)\right)$,

where $I_{K}$ is the minimum of $I(\mathbf{y}, \mathbf{x})$, for $\mathbf{x} \notin K$ and for $\mathbf{y} \in K$.

This result follows, like Theorem 2, from the fact that each $\mathrm{C}_{i}$ can be approximated by a Poisson distributed $\mathrm{U}_{i}$, conditioned on the $\bigcup_{i}$ 's summing to $n \mathbf{x}$. The $o(1)$ is an adjustment to account for the conditioning (see appendix for further notes on Cramér's theorem and its application).

For most biologically reasonably models, $I_{K}=$ $I\left(\mathbf{x}_{b}, \mathbf{x}_{b}\right)$, where $\mathbf{x}_{b}$ lies on the boundary of the closed set $K$. Thus, the bound is determined by the rate at which points on the boundary of $K$ remain on the boundary. The resultant bound on the probability is likely to be, relative to the real extinction probability, extremely high. Indeed, the bound is not intended as an accurate estimate of extinction probability and instead merely demonstrates that probability of extinction is exponentially small in the number of sites, $n$. While the normal approximation in Theorem 2 gives the 'stable' population distribution away from extinction, Theorem 4 tells us then the time till extinction is reached grows exponentially with the number of resource sites.

Obtaining accurate estimates of the real extinction probability for a particular site model remains a difficult problem. Since $I$ is a rate equation, we might suppose that the expected time until the population enters a set $K$ from a starting point, $\mathbf{X}_{t}=\mathbf{x}$ is simply $1 / I\left(\mathbf{x}_{b}, \mathbf{x}\right)$, where $\mathbf{x}_{b}$ is on the boundary of $K$. However, even if $\mathbf{X}_{t+1} \notin K$ it is not reasonable to assume that $\mathbf{X}_{t+1}=\mathbf{x}$. Indeed, $\mathbf{X}_{t}$ changes on every generation, and so then does $I\left(\mathbf{x}_{b}, \mathbf{X}_{t}\right)$. Furthermore, extinction may occur through two or more 'large deviations' and it is only by looking at how a sequence of such deviations might occur that we can understand the extinction probability.

Despite the above observations, we now give some heuristic discussion of how extinction time might be estimated for a site model with a stable fixed point, $\mathbf{x}=\mathbf{x}_{*}$, of Eq. (5). We might assume that $\mathbf{X}_{t+\tau}=\mathbf{x}$ for all $\tau<T_{e}$ up until $\mathbf{X}_{t+T_{e}} \in K$. An estimate for the time, $T_{e}$, at which the population enters $K$ through a large deviation is then $\widehat{T_{e}}=\exp \left(n I\left(\mathbf{x}_{b}, \mathbf{x}_{*}\right)\right)$.

This estimate assumes that a single transition takes the system away from the attracting fixed point, directly into $K$. An extension of this, for a single species with $K=\left[0, x_{b}\right]$, which attempts to account for the 'normal' fluctuations around $x_{*}$ is

$$
\begin{aligned}
1 / \widehat{T_{e}}= & \frac{1}{\sqrt{2 w\left(x_{*}\right) \pi}} \int_{x=x_{b}}^{\infty} \exp \left(-\frac{\left(x_{*}-x\right)^{2}}{2 w\left(x_{*}\right)}\right) \\
& \times \exp \left(-n I\left(x_{b}, x\right)\right) d x,
\end{aligned}
$$

where $w\left(x_{*}\right)$ is as defined in Eq. (11). While possibly incorporating the distribution of $x$ before the population enters $K$ this estimate still fails to account for the fact that for the population to enter $K$ may take two or more large deviations. We discuss these estimates further in the context of a particular interaction function later in this paper.

\section{Single species competition}

The applicability of site-based models and the above results can only be made clear through examples and the rest of this paper is dedicated to presenting examples where Proposition 1, Theorems 2 and 4 can be applied. Such a presentation serves not only to illustrate the application of the theorems, but also aid in understanding the proof of the theorems given in the appendix. We begin by presenting a contest competition model, by first describing the model and deriving a density approximation without reference to the general site-based models of the previous section. In doing so, we aim to highlight the logic of our approach, and thus illustrate how the site-based framework may be applied to specific models.

\subsection{Contest competition}

Consider the following description as the basis of a site-based model of a contest for breeding sites 
(Nicholson, 1954). On generation $t, M_{t}$ animals choose one of $n$ sites uniformly at random. If, after all the animals are placed at the sites, a particular site contains only one animal, then this animal produces $b$ offspring. If two or more animals choose the same site then only one of these animals can successfully reproduce. We choose the successful animal uniformly at random amongst all the animals at the site. We also assume that reproducing animals die, leaving only $b$ offspring at the site. Between breeding stages, the animals have a constant probability, $p$, of surviving to the next stage of reproduction. Fig. 1 illustrates how such contest competition works for three generations with $n=10$ sites.

The population density, relative to the number of sites $n=100$, for a computer simulation of a contest model for various values of $b$ is shown in Fig. 2. From the simulations it appears that provided $b>1 / p$ the population seems to settle at a stable level. While it is possible to use such simulations to determine the equilibrium population for particular parameter values, the simulations do not provide a mathematical link between the individual-level parameters and the population dynamics. Ideally, we would like to plot the equilibrium population - if indeed such an equilibrium does always exist - as a function of $b$ and $p$, without running simulations repeatedly for different parameter values.

To understand the relationship between population dynamics and the individual level parameters we derive a simple mean approximation for our stochastic sitebased model. The conditional expectation of the population at the next generation, $M_{t+1}$ given that the current population $M_{t}=m$, is the sum of the expected number of offspring produced each year multiplied by their probability of survival until the next year:

$$
\begin{aligned}
\mathbf{E}\left(M_{t+1} \mid M_{t}=m\right)= & p \sum_{s=1}^{n} \mathbf{E}(\text { no. of offspring } \\
& \text { produced at site } \left.s \mid M_{t}=m\right) .
\end{aligned}
$$

By introducing the observation that sites which do not contain an animal produce no offspring, while sites containing one or more animals produce $b$ offspring we get

$$
\begin{aligned}
\mathbf{E}\left(M_{t+1} \mid M_{t}=m\right)= & p \sum_{s=1}^{n} b \mathbf{P}(\text { at least one animal } \\
& \text { at site } \left.s \mid M_{t}=m\right) .
\end{aligned}
$$

Since the animals are distributed uniformly at random between $n$ cells we can write

$$
\begin{aligned}
\mathbf{E}\left(M_{t+1} \mid M_{t}=m\right) & =b p \sum_{s=1}^{n}\left(1-(1-1 / n)^{m}\right) \\
& =\rho n\left(1-(1-1 / n)^{m}\right),
\end{aligned}
$$

where we can think of $\rho=b p$ as the density-independent reproductive gain per individual. The fact that $1-(1-$ $1 / n)^{m} \leqslant 1$ ensures that the expected population $\mathbf{E}\left(M_{t+1} \mid M_{t}=m\right)$ will never exceed $\rho n$.

By noting that $\ln (1-1 / n)+1 / n \rightarrow 0$ very quickly as $n \rightarrow \infty$ we see that for large $n$,

$\mathbf{E}\left(M_{t+1} \mid M_{t}=m\right)=\rho n\left(1-e^{-m / n}\right)$.

By iterating this expectation we can find an approximation for the evolution of the population density, $X_{t}=$ $M_{t} / n$, of individuals:

$x_{t+1}=f\left(x_{t}\right):=\rho\left(1-e^{-x_{t}}\right)$.

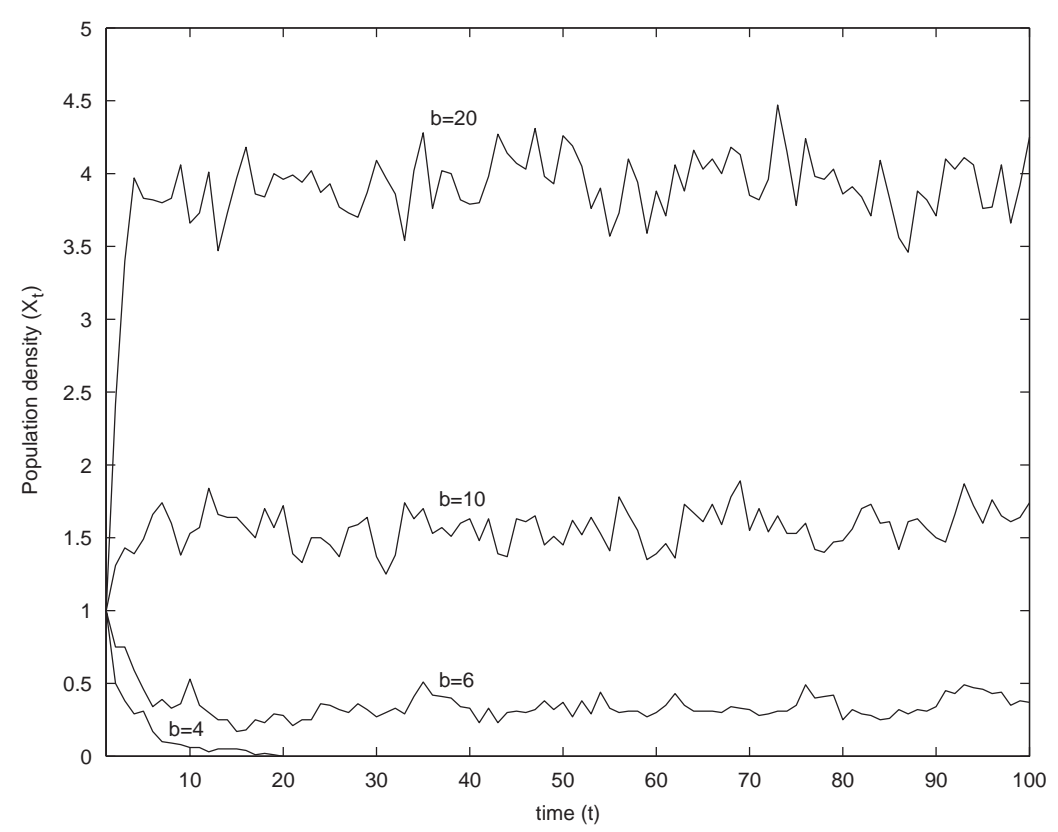

Fig. 2. The population density $M_{t} / n$, for a computer simulation of a contest model with $n=100, p=0.2$ and initial population $M_{0}=100$, for $b$ equal to $4,6,10$ and 20 . 
The map $f$ has a fixed point at 0 and the set $[0, \rho]$ is invariant under its action. To ascertain the local stability of 0 , we note that the gradient of $f(x)$ is $f^{\prime}(x)=\rho e^{-x}$. Thus, $f^{\prime}(0)>1$ provided the density-independent reproductive gain, $\rho>1$ the population will grow. When $\rho<1$ populations will decrease to zero.

If a population of one animal will grow (i.e. $\rho>1$ ) then $f$ has exactly one non-zero fixed point which is stable. Thus, the population stabilises at $x_{*}$, the nonzero solution to $\ln (1-x / \rho)=x . x_{*}$ accurately approximates the mean population density of the site-based simulations. For example, when $b=6$ and $p=0.2$, the attracting fixed point of $f$ is $x_{*}=1.594$, which can be compared to that for the simulation in Fig. 2 where the population density for $b=6$ and $n=100$ was on average 1.589. In general, $x_{*}$ lies within $1 / n$ of the simulated mean. Eq. (18) thus gives a very accurate description of the dynamics of our site-based model.

In the preceding discussion we have shown how a density approximation can be derived for the specific site-based contest model, without reference to the more general site-based models presented in the previous section. Contest competition may, however, be described using the following interaction function:

$\phi\left(C_{i}, \xi_{i}\right)= \begin{cases}\xi_{i}, & C_{i}>0 \\ 0, & C_{i}=0,\end{cases}$

where $\xi_{i} \sim \operatorname{Bin}(b, p)$. Then applying the density approximation in Proposition 1 directly we get precisely the same density approximation as in Eq. (18). Furthermore, we can now employ the normal approximation in Theorem 2, to obtain the population variance associated with $\phi$,

$$
\begin{aligned}
v(x)= & \operatorname{Var}[\phi]-x\left(\frac{d f}{d x}\right)^{2} \\
= & \left(\rho(1-p)+\rho^{2}\right)\left(1-e^{-x}\right) \\
& -\left(\rho\left(1-e^{-x}\right)\right)^{2}-x\left(\rho e^{-x}\right)^{2} \\
= & \rho(1-p)\left(1-e^{-x}\right)+\rho^{2} e^{-x}\left(1-(1+x) e^{-x}\right) .
\end{aligned}
$$

Using Eq. (9), the dynamics of our site-based model are thus approximated by the stochastic equation

$x_{t+1}=\rho\left(1-e^{-x_{t}}\right)+\sqrt{\frac{v\left(x_{t}\right)}{n}} \varepsilon_{t}$,

where each $\varepsilon_{t} \sim N(0,1)$.

The equilibrium distribution of Eq. (21) can now be solved, and is very close to that of simulations of the original site-based model. Indeed, Fig. 3 compares, for various values of $b$, the standard deviation around the equilibrium distribution of the site-based contest model, with the standard deviation estimated using Corollary 3 :

$$
\begin{aligned}
& w\left(x_{*}\right) \\
& =\frac{\rho(1-p)\left(1-e^{-x_{*}}\right)+\rho^{2} e^{-x_{*}}\left(1-\left(1+x_{*}\right) e^{-x_{*}}\right)}{\left(1-\left(\rho e^{-x_{*}}\right)^{2}\right)} .
\end{aligned}
$$

This standard deviation estimate is within $1 / n$ of the simulation variance for all values of $b$. The normal approximation can thus produce extremely accurate descriptions of the equilibrium distribution of our sitebased model, even when $n=100$ is relatively small. In general, for interaction functions where the underlying density approximation has a single stable fixed point, both the mean and the variance can be estimated with an error of only $1 / n$ using Proposition 1 and Corollary 3, respectively. It is thus possible to take the rules governing interaction between individuals and give accurate analytic approximations of the distribution of the population over time.

\subsection{Scramble competition}

In contest competition, one winner takes all of the resource no matter how many individuals compete for that resource. Another equally plausible mechanism for competition, known as scramble competition, is where overcrowding of a particular resource can lead to neither individual benefiting from the resource (Nicholson, 1954). Consider the following model of scramble competition, where if two or more individuals compete for the same resource site, none of them produce any offspring. Individuals occupying a site on their own produce $b$ offspring, which survive with probability $p$. The interaction function for this process is thus

$\phi\left(C_{i}, \xi_{i}\right)= \begin{cases}\xi_{i}, & C_{i}=1, \\ 0, & \text { otherwise }\end{cases}$

where $\xi_{i} \sim \operatorname{Bin}(b, p)$.

The density approximation of the interaction function in Eq. (23) is the exponential logistic or Ricker map:

$x_{t+1}=\rho x_{t} e^{-x_{t}}$,

where, as before, $\rho=b p$. The non-zero equilibrium density of individuals is thus the non-zero fixed point of Eq. (24), $x_{*}=\ln (\rho)$. Unlike contest competition, where the population always reached a stable level, for scramble competition if $\ln (\rho)>2$ then the population will have periodic dynamics and, for even larger values of $\rho$, chaotic dynamics (Sumpter and Broomhead, 2001).

Using Theorem 2, the estimated variance of the scramble site model defined by (23) is

$$
\begin{aligned}
v\left(x_{t}\right)= & \rho(1-p) x_{t} e^{-x_{t}} \\
& +\rho^{2} x_{t} e^{-x_{t}}\left(1-\left(1-x_{t}+x_{t}^{2}\right) e^{-x_{t}}\right) .
\end{aligned}
$$

Fig. 4 compares the distribution of population densities of the site-based scramble model with that of iterations of the stochastic difference equation

$x_{t}=\rho x_{t} e^{-x_{t}}+\sqrt{\frac{v\left(x_{t}\right)}{n}} \varepsilon_{t}$,

where each $\varepsilon_{t} \sim N(0,1)$. The three different parameter values shown in Fig. 4 correspond to population 




Fig. 3. The standard deviation around the equilibrium distribution of the site-based model (crosses), taken over 5000 generations, compared to the prediction in Eq. (22), $\sqrt{w\left(x_{*}\right) / n}$ (solid line). Here $n=100$ and $p=0.2$.

dynamics with a stable fixed point $(\rho=6)$, a period-2 cycle $(\rho=12)$ and chaotic oscillations $(\rho=18)$. Provided the population does not become extinct, iteration of Eq. (26) reproduces the distribution of the site-based simulation extremely well.

\subsection{Competition models from first principles}

The density approximation can give important insight into the theoretical justification of particular models. In the preceding discussion we have shown how the dynamics of scramble competition are captured by the Ricker map and contest competition by a saturating function with no turning point. Inverting Eq. (3), the density approximation allows us to infer the interaction rules implied by other difference equations. For example, the logistic equation $x_{t+1}=r x_{t}\left(1-x_{t}\right)$ is often cited as a simple example of a model of population dynamics but less often fit to biological data, since it can, for $r>4$, produce negative populations. It is interesting to note then

$$
\begin{aligned}
\Phi(x) & =e^{-x} e^{x} r x_{t}\left(1-x_{t}\right) \\
& =e^{-x}\left(r x-\frac{3 r x^{3}}{3 !}-\frac{8 x^{4}}{4 !}-\frac{15 x^{5}}{5 !}-\cdots\right) .
\end{aligned}
$$

Equating co-efficients to Eq. (3) gives an interaction function

$\phi\left(C_{i}, \xi_{i}\right)=-r C_{i}\left(C_{i}-2\right)$

which for all $C_{i}>2$ gives negative populations at a resource site (i.e. each animal can give birth to a negative number of offspring). There is thus little biological justification for using the logistic equation as a model of population dynamics when individuals are known to reproduce after being distributed at random between discrete resource sites. In this way, the density approximation can be used to make explicit and test the assumptions implied about animal interactions when fitting difference equation models to field data.

\subsection{Evolutionary stable strategies: scramble vs. contest}

While fitting interaction functions to data addresses problems in applied ecology, a theoretical question that often arises when considering contest and scramble competition concerns which type of competition is most advantageous to the reproducing individual. In our model, a population of individuals employing the contest strategy are able to maintain a larger population than those employing the scramble strategy: the contest strategy gives an equilibrium population density close to $\rho$, while, if the scramble site model has a stable population density it is equal to $\ln (\rho)$. In this sense, the carrying capacity of the environment is dependent on the interaction of the individuals. The fact that contest gives a much larger population size does not however imply that, given a choice of contest or scramble strategies, contest is optimal for an individual. To find the optimal or evolutionary stable strategy we must consider interactions between contest and scramble individuals.

Scramble individuals could be thought of as using the resource either by exploiting it without regard for its limited capacity: i.e. they adopt an aggressive behaviour toward the resource which renders it unusable by any other individual. To model this concept, we assume that 

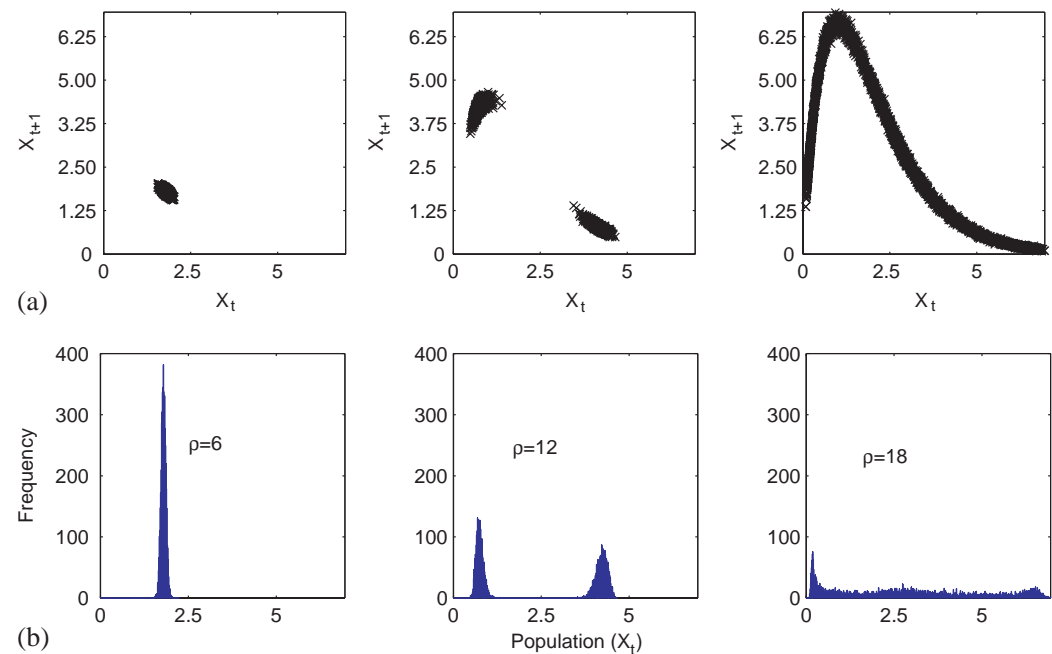

(b)
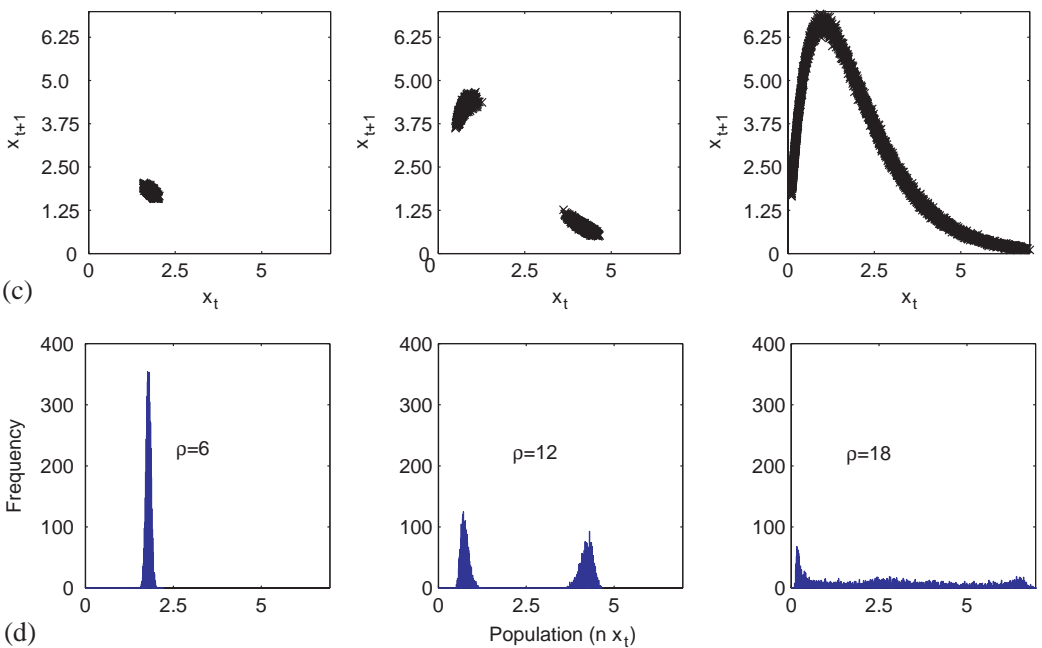

Fig. 4. Comparison between equilibrium population density distribution of (a,b) a site-based simulation and (c, d) iteration of Eq. (26) for $n=4000$ and $\rho=6$ (a stable fixed point), $\rho=12$ (oscillations with period two) and $\rho=18$ (chaotic oscillations). (a) and (c) show the population on generation $t$ plotted against the resultant population on generation $t+1$. (b) and (d) show frequency distributions. Both these plots are generated over 5000 generations starting from the 1000th generation.

a single scramble individual produces all the offspring when at a resource site on its own or with only contest individuals, but when at a site with one or more other scramble individuals no offspring are produced. Contest individuals produce no offspring whenever one or more scramble individuals are present at a resource site. If only contest individuals are present at a site then, as in our original contest model, $\xi_{i} \sim \operatorname{Bin}(b, p)$ offspring are produced by one randomly chosen individual.

These assumptions give an interaction function as follows:

$$
\begin{aligned}
\phi & \left(\left(C_{i, 1}, C_{i, 2}\right), \xi_{i}\right) \\
\quad & \begin{cases}\left(\xi_{i}, 0\right), & C_{i, 1}>0 \text { and } C_{i, 2}=0, \\
\left(0, \xi_{i}\right), & C_{i, 1}=0 \text { and } C_{i, 2}=1, \\
(0,0), & \text { otherwise, }\end{cases}
\end{aligned}
$$

where $C_{i, 1}$ and $C_{i, 2}$ are, respectively, the number of contest and scramble individuals at site $i$. In terms of evolutionary game theory $\phi$ can be thought of as a payoff function for the two strategies. Note that in this case $\xi_{i}$ is the same for scramble and contest individuals, i.e. there is no cost to be paid by being a scramble individual.

As with the separate contest and scramble models, we can derive an approximation of our combined contest and scramble model. Applying Proposition 1 directly we get

$$
\left(\begin{array}{c}
x_{t+1} \\
y_{t+1}
\end{array}\right)=\Phi\left(\begin{array}{c}
x_{t} \\
y_{t}
\end{array}\right)=\left(\begin{array}{c}
\rho\left(1-e^{-x_{t}}\right) e^{-y_{t}} \\
\rho y_{t} e^{-y_{t}}
\end{array}\right)
$$

for the evolution of the contest $\left(x_{t}\right)$ and scramble $\left(y_{t}\right)$ populations, respectively. We can see immediately that the change in the number of scramble individuals does not depend on the number of contest individuals, and indeed equation for $y_{t+1}$ is equivalent to Eq. (24). Eq. (28) shows, however, that the rate of growth of contest individuals is always reduced by the inclusion 
of scramble individuals in the population. Furthermore, when the scramble population reaches equilibrium at $y_{*}=\ln (\rho)$ :

$x_{t+1}=\rho\left(1-e^{-x_{t} / n}\right) e^{-\ln (\rho)}=1-e^{-x_{t}}$.

Differentiating the right-hand side with respect to $x_{t}$ we get $e^{-x_{t}}<1$ and hence $x_{t+1}<x_{t}$ for all $x_{t}>0$. Thus, at an equilibrium population of scramble individuals, the population of contesters always decreases. At small populations of contesters and scramblers both populations will grow, but provided $\rho$ is in the range of values at which $y_{t}$ goes to a positive stable equilibrium, the scramble individuals will eventually cause the contest individuals to become extinct. A similar result holds for the case when Eq. (28) has periodic and chaotic cycles, contesters will eventually become extinct (result due to J. Stark, personal communication).

The fact that scramble is selected for has consequences for the dynamics of the population. Scramble populations may have periodic or chaotic dynamics, while contest populations will always have stable dynamics. The large fluctuations in the population that occur when the dynamics are unstable can result in extinction of the population. The problem is compounded by the fact that large values of $\rho$ are also selected for: if two scramble populations with different values of $\rho$ compete with each other, the population with the smaller value of $\rho$ will eventually go extinct. Thus, in our model, chaotic population dynamics and increased rates of population extinction are actually selected for. At the level of the individual, scramble strategy and large $\rho$, which are the conditions for chaos at a population level, are the evolutionary stable strategy. Indeed, our model results in a chaotic 'tragedy of the commons': the contest strategy which maximised resource usage is replaced by a scramble strategy which at best greatly reduces resource usage and at worse leads to population extinction.

\section{Other site-based models}

\subsection{Co-operation}

The application of the density and normal approximations is not limited to questions of competition for resource sites. We can also consider situations where some form co-operation is required for offspring to be produced at a resource site. For example, if two or more individuals are needed per resource site in order for offspring to be produced then the interaction function is

$\phi\left(C_{i}, \xi_{i}\right)= \begin{cases}\xi_{i}, & C_{i}>1, \\ 0, & \text { otherwise },\end{cases}$

where, as before, $\xi_{i} \sim \operatorname{Bin}(b, p)$ and $\rho=E\left[\xi_{i}\right]=b p$. Using Proposition 1 , the density approximation is thus $x_{t+1}=h\left(x_{t}\right)=\rho\left(1-\left(1+x_{t}\right) e^{-x_{t}}\right)$.

Cobweb diagrams for two qualitatively different parameterisations of Eq. (30) are shown in Fig. 5.

The function $h$ has a fixed point at $h(0)=0$. The fact that $h^{\prime}(0)=0$ indicates that 0 is a turning point. Furthermore, as $x \rightarrow \infty, h(x) \rightarrow \rho$ and $h(x)$ has a point of inflection at 1 , where the shape of $h$ changes from convex to concave. For small values of $\rho, h\left(x_{t}\right)$ will fail to cross the line $x_{t+1}=x_{t}$ (see Fig. 5a), but as $\rho$ is increased, a saddle node bifurcation occurs and two fixed points (one stable and one unstable) appear (see Fig. 5b). The new stable fixed point corresponds to a sustainable positive population. An initial population greater than the unstable fixed point will grow to a level equal to the position of the stable fixed point, while an initial population less than the unstable fixed point will die out to zero. Solving numerically $x=h(x)$ for $\rho$ gives the bifurcation point, having value $\rho=3.351$, at which a stable population is possible. Thus, individuals must on average produce at least 3.351 offspring which survive to the next generation in order to have a non-zero sustainable population.

When $\rho>3.351, h$ has three fixed points: a stable point $x_{*}=0$ corresponding to extinction, an unstable point $x_{*}=u$ corresponding to the minimum sustainable population and a stable point $x_{*}=s$ corresponding to a positive stable population. Given the site-based model with a population fluctuating around the positive stable equilibrium, we can use the large deviation theory (Theorem 4) to predict time until till extinction for various $\rho$. From Eq. (12), the logarithmic moment generating function of $h$ is

$\Lambda(\beta, x)=\log \left\{e^{\beta \rho} e^{x}-\left(e^{\beta \rho}-1\right)(1+x)\right\}-x$

and we can solve Eq. (14) for $\beta$ to get

$$
\begin{aligned}
I(y, x)= & -\log \left(\frac{\rho-y}{\rho e^{-x}(1+x)}\right) \\
& -\frac{y}{\rho} \log \left(\frac{y(1+x)}{(\rho-y)\left(e^{x}-1-x\right)}\right)
\end{aligned}
$$

the rate function of $h$.

Fig. 6 shows time till extinction for the co-operation model starting with a population of $n b p$, where $n=100$, $p=0.01$ and $b$ ranges from 335 to 363 (i.e. $\rho$ ranges from 3.35 to 3.63 ). The crosses show the mean time till extinction for 100 simulations, for each parameter value, of the site-based model. The solid and dotted lines show various bounds and estimates on the probability of extinction and estimates on the time till extinction. The extinction time for the site model simulations grow exponentially with $b$. Once $\rho$ exceeds 3.63, each simulation of the site model takes over a day to execute on a fast desktop computer.

We now describe in detail where the bounds and estimates of extinction time shown in Fig. 6 are 

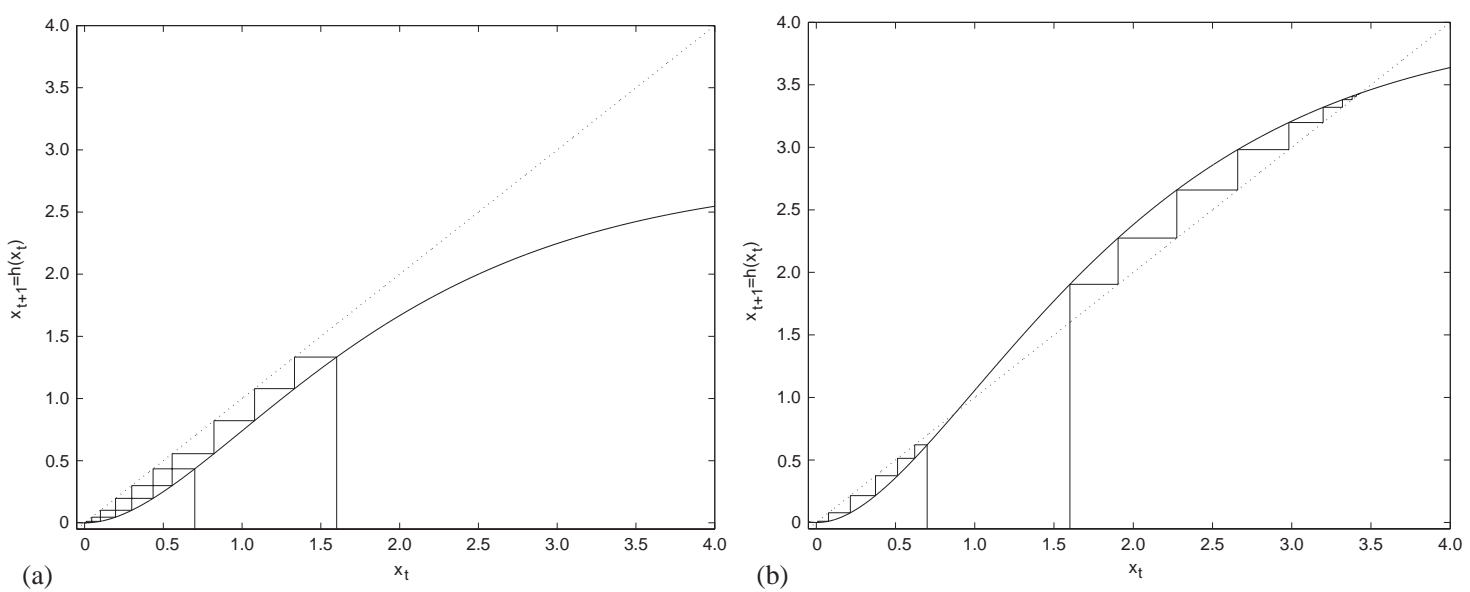

Fig. 5. The cobweb diagram for the map $h$ given in Eq. (30) for (a) $b=7$ and (b) $b=10$. In both cases $p=0.4$. The 'cobweb' shows how a starting population density of 0.65 or 1.55 either increase or decrease through repeated iterations of the function $h$.

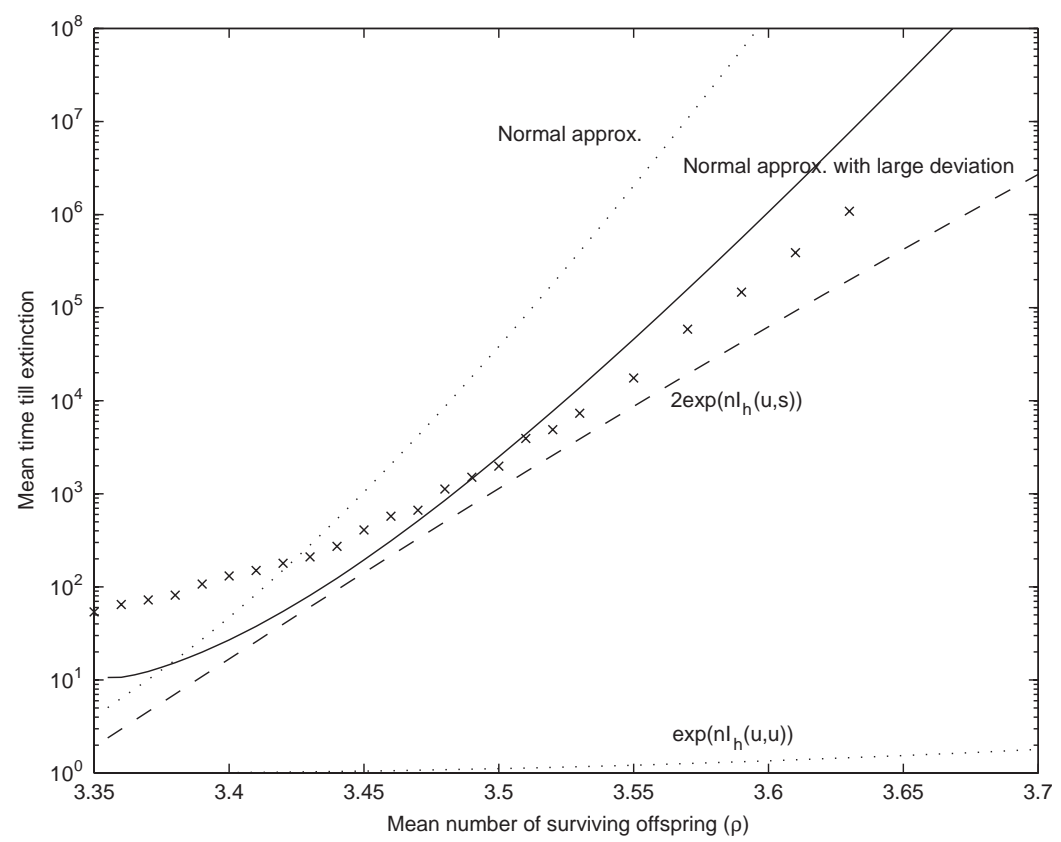

Fig. 6. Extinction times for the site-based model of co-operation. The crosses represent extinction times average extinction times over 100 simulations for a starting population of $n b p$ in a site-based model with interaction function 29. In all simulations, $n=100$ and $p=0.01 . b$ goes from 335 to 363 , so that $\rho$ goes from 3.35 to 3.63 . The lower dotted line is the minimum bound for extinction time given in 32 . The solid and dashed lines are based on large deviation estimates (see text for details). The upper dotted line is the estimate obtained by the integral in Eq. (34).

obtained. Recall, from Eq. (15) that we can calculate an absolute upper bound for the probability of extinction by defining a set $K$ that contains 0 but does not contain $s$. A natural candidate for this set is $K=[0, u]$, since below $u$ the population will 'usually' go to 0 and above $u$ the population will 'usually' go toward $s$. The dotted line at the bottom of Fig. 6 is

$\exp (n I(u, u))$

the lower bound on extinction time. Clearly, this bound lies a long way from the actual extinction time of the site-based simulations but it serves to prove that extinction time does indeed grow exponentially with the number of sites $n$.

The dashed and solid lines in the middle part of Fig. 6 are estimated extinction times using our heuristics in Eqs. (16) and (17), respectively. The dashed line is derived from Eq. (16),

$2 \exp (n I(u, s))$

which is twice the rate at which $s$ goes $u$. The multiplication by a factor of 2 comes from the fact that when the population is at exactly the minimum sustainable level it will, with a probability of approxi- 
mately $1 / 2$, move toward extinction and otherwise with a probability of approximately $1 / 2$ move toward the positive stable equilibrium. The solid line is an estimate based on evaluating the integral in Eq. (17), for the appropriate $w(s)$ and $I(u, s)$ functions. This estimate attempts to account for the 'normal' fluctuations around $\mathbf{x}_{*}$ as well as large deviations by evaluating the integral in Eq. (17), for the appropriate $w(s)$ and $I(u, s)$ functions. Both of these estimates lie nearer to the mean extinction times from the simulations than the lower bound, but neither accurately captures the shape of the curve as $b$ increases.

The estimates obtained from Eqs. (16) and (17) are however more accurate than that obtained by the normal approximation. The dotted line toward the top of Fig. 6 shows an extinction probability estimated by numerically integrating

$1-\frac{1}{\sqrt{2 w(s) \pi}} \int_{x=u}^{\infty} \exp \left(-\frac{(s-x)^{2}}{2 w(s)}\right) d x$

where $w(s)$ is calculated using Eq. (11). This approximation greatly over-estimates extinction time as $b$ increases, and contrasts strongly with the very good approximation provided by the normal approximation in the 'short' term: as evidenced in Figs. 3 and 4. While the central area of the normal distribution and the distribution generated by site-based models are very similar, the tails of these two distributions are very different. Thus, although the normal approximation gives a very good estimate of the 'normal' behaviour of the population dynamics of site-based models while the population remains away from extinction the approximation is very poor at predicting when that extinction will occur. Large deviation theory may be used to produce better estimates, and we have suggested two candidates, but more research is required in this area. In particular, we need to establish if it really is a single large deviation, as assumed in Eq. (33) or a number of deviations which produce extinctions.

\subsection{Predator-prey dynamics}

There are a number of ways in which interactions between predators and prey may be incorporated into an interaction function. A simple example is as follows:

$$
\phi\left(\begin{array}{ll}
\left.\left(C_{1, i}, C_{2, i}\right),\left(\xi_{1, i}, \xi_{2, i}, R_{i}\right)\right) \\
=\left\{\begin{array}{cc}
(0,0), & C_{1, i}=0 \text { and } C_{2, i}=0, \\
\left(C_{1, i} \xi_{1, i}, 0\right), & C_{1, i}>0 \text { and } C_{2, i}=0, \\
\left(0, C_{2, i} \xi_{2, i}\right), & C_{1, i}>0 \text { and } C_{2, i}>0, \\
\left(0, C_{2, i} R_{i}\right), & C_{1, i}=0 \text { and } C_{2, i}>0,
\end{array}\right.
\end{array}\right.
$$

where $C_{1, i}$ and $C_{2, i}$ are, respectively, the number of prey and predators at site $i$. Here, the prey will reproduce without limit in the absence of predators, producing $\xi_{1, i} \sim \operatorname{Bin}(a, p)$ offspring each generation. However, if the prey occupies the same resource site as a predator then all predators at that site produce $\xi_{2, i} \sim \operatorname{Bin}(b, q)$ offspring. Predators landing on sites without prey will only survive with probability $r$, so that $R_{i}$ is Bernoulli distributed with parameter $r$.

The density approximation for the interaction function in Eq. (35) is given by

$$
\left(\begin{array}{c}
x_{t+1} \\
y_{t+1}
\end{array}\right)=\left(\begin{array}{c}
\alpha x_{t} e^{-y_{t}} \\
y_{t}\left(r e^{-x_{t}}+\beta\left(1-e^{-x_{t}}\right)\right)
\end{array}\right)
$$

where $\alpha=E\left[\xi_{1, i}\right]=p a$ and $\beta=E\left[\xi_{2, i}\right]=q b$. These equations have fixed points at $x_{*}=y_{*}=0$ and $x_{*}=$ $\ln ((\beta-r) /(\beta-1))$ and $y_{*}=\ln (\alpha)$. The eigenvalues of the Jacobian of $\left(y_{t+1}, x_{t+1}\right)$ at these fixed points are $\alpha$ and $r$ for the zero fixed point and $1 \pm \sqrt{x_{*} y_{*}(1-\beta)}$ for the non-zero fixed point. Thus, provided, $\alpha>1$ the zero fixed point is unstable. Furthermore, since the non-zero fixed point either has one eigenvalue greater than unity (for $\beta<1$ ) or a pair of complex eigenvalues with a modulus greater than unity (for $\beta>1$ ), it is also unstable. This observation implies unlimited growth of either prey alone or both predators and prey together. However, for finite $n$ there cannot be unlimited growth of both prey and predators, since once predator numbers are sufficiently large they will occupy every resource site and kill every prey. Thus, predators will always become extinct.

A small adjustment to the interaction function completely changes the predator-prey dynamics. We use the interaction function

$$
\begin{aligned}
\phi & \left(\left(C_{1, i}, C_{2, i}\right),\left(\xi_{1, i}, \xi_{2, i}, R_{i}\right)\right) \\
= & \left\{\begin{array}{cc}
(0,0) & C_{1, i} \neq 1 \text { and } C_{2, i}=0, \\
\left(\xi_{1, i}, 0\right) & C_{1, i}=1 \text { and } C_{2, i}=0, \\
\left(0, C_{2, i} \xi_{2, i}\right) & C_{1, i}>0 \text { and } C_{2, i}>0, \\
\left(0, C_{2, i} R_{i}\right) & C_{1, i}=0 \text { and } C_{2, i}>0,
\end{array}\right.
\end{aligned}
$$

so that prey have scramble competition in the absence of predators. The density approximation becomes

$$
\left(\begin{array}{l}
x_{t+1} \\
y_{t+1}
\end{array}\right)=\mathbf{g}\left(x_{t}, y_{t}\right):=\left(\begin{array}{c}
\alpha x_{t} e^{-x_{t}} e^{-y_{t}} \\
y_{t}\left(r e^{-x_{t}}+\beta\left(1-e^{-x_{t}}\right)\right)
\end{array}\right) .
$$

These equations now have three non-negative fixed points: at $(0,0)$ corresponding to extinction of both predators and prey; at $(\ln (\alpha), 0)$ corresponding to predator extinction and the prey reaching the carrying capacity for scramble competition; and at

$$
\left(\ln \left(\frac{-r+\beta}{\beta-1}\right), \quad \ln \left(\frac{\alpha(\beta-1)}{\beta-r}\right)\right),
$$

where predator and prey co-exist. Dependent on the values of $\alpha, \beta$ and $r$, iteration of Eq. (38) will either move to one of these fixed points, enter a stable cycle or (possibly) oscillate chaotically.

Fig. 7(a) shows a simulation of the site-based model defined by the interaction function in Eq. (37) compared 

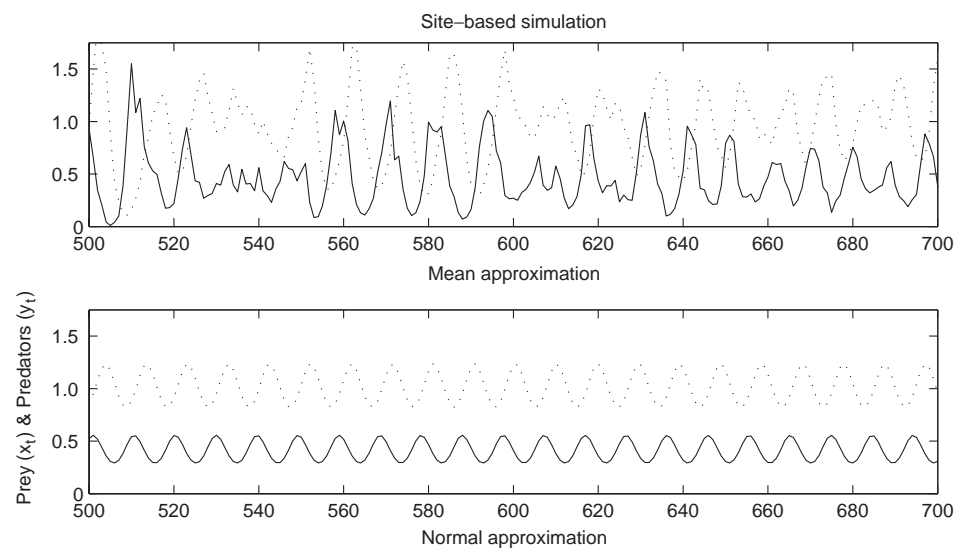

(a)
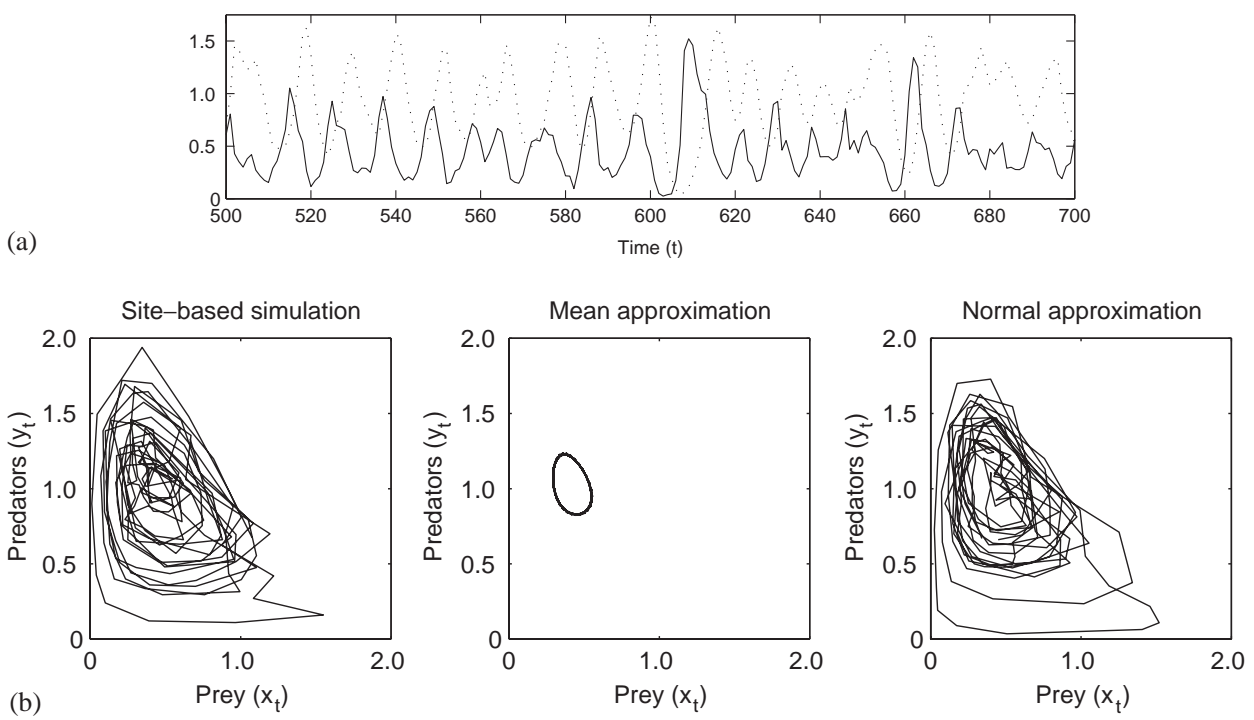

Fig. 7. Comparison of a simulation of the site-based model defined by the interaction function in Eq. (37), a numerical solution to Eq. (38) and a numerical solution of the normal approximation of the site-based model with noise determined by the variance matrix in equation. Solid lines are prey population densities and dashed lines are predator population densities. Plot (a) is the results in time series form, while (b) is the same data plotted as an implicit prey vs. predator form. In all simulations/solutions $a=7, b=4, p=0.6, q=0.5$ and $r=0.5$.

to a numerical solution to Eq. (38) and a numerical solution of the normal approximation of the site-based model. Fig. 7(b) shows the same data plotted as prey population against predator population. The noise term in for the normal approximation, when there are $x$ prey and $y$ predators, is calculated from the covariance matrix

$$
\begin{aligned}
v(\mathbf{x})= & \left(\begin{array}{c}
\alpha((1-p)+\alpha) x e^{-x-y} \\
y\left(r((1-r)+1) e^{-x}+\beta((1-q)+\beta)\left(1-e^{-x}\right)\right)
\end{array}\right) \\
& \times\left(\begin{array}{ll}
1 & 0 \\
0 & 1
\end{array}\right) \\
& -\mathbf{g}(x, y) \mathbf{g}(x, y)^{\mathrm{T}}-\mathbf{J}\left(\begin{array}{ll}
x & 0 \\
0 & y
\end{array}\right) \mathbf{J}^{\mathrm{T}}
\end{aligned}
$$

where

$\mathbf{J}=\left(\begin{array}{cc}\alpha(1-x) e^{-x-y} & -\alpha x e^{-x-y} \\ y(\beta-r) e^{-x} & r e^{-x}+\beta\left(1-e^{-x}\right)\end{array}\right)$

is the Jacobian of $\mathbf{g}$.
The mean approximation model alone proves to be a poor approximation of the site-based model. This can be seen in the differences in the time series between the mean approximation and the site-based simulations in Fig. 7. The normal approximation provides a much better match to the site-based model. Indeed, tested over 100 simulations, the difference between site-based simulations and normal approximation in the mean and variance was seen to be less than $1 / n$. Furthermore, in all of these 100 site-based and normal approximation simulations both populations went extinct, with an average extinction time over 100 simulations of 1042 time steps for the site-based model and 975 for the normal approximation. This accurate reproduction of the dynamics of the site-based model by the normal approximation is seen to hold for a whole range of parameter values, even when $n=200$ is relatively small.

The difference between our first and second predatorprey models illustrates an important point about interaction functions: seemingly small changes in the 
interaction rules can produce rather large changes in the dynamics of the population. In this sense, the interaction functions we have considered here are not structurally stable: the outcome in terms of population dynamics is highly dependent on the structure of the rules. The question then is how well these structural dependencies are mimicked by real animal populations. For example, are there circumstances in which removing density dependence of prey can lead to both prey and predator extinction? The power of the interaction function and the application of Proposition 1 is that the consequences of changes in the interactions of different species can be easily examined. An interesting area of future research would be a systematic comparison of different predatorprey models both in terms of local interactions and population dynamics.

\section{Discussion and future directions}

In this paper, we have presented a methodology for understanding the dynamics of a whole range of ecological systems that can be described in terms of site-based interactions between individuals. The methodology is grounded in three main results: the density approximation (Proposition 1), the normal approximation (Theorem 2) and a large deviation theory (Theorem 4). The density approximation demonstrates a large system size equivalence between site-based models and difference equations. The normal approximation gives the variance in this approximation and allows approximation of site-based models even for relatively small systems with complicated dynamics. The large deviation theorem gives bounds and estimates on extinction probability, not captured by the normal approximation. The intention of this paper has been to lay a foundation by stating, proving and illustrating these results. The main challenge to our results is whether they are applicable to understanding real ecological systems. As a conclusion, we discuss how our models might be fitted to field data, along with some ideas on how problems of spatial scale and local dispersal might be addressed. Finally, we note a role that site-based models may have in understanding evolutionary dynamics.

\subsection{Fitting models to field data}

In order to predict future change in animal populations difference equation models can be fitted to time series data of past observations of these populations. Such fitting is commonly made by examining the yearon-year density dependent reproduction or using other general methods for the study of non-linear time series (see, for a review, Berryman, 1999). One of the major limitations of such an approach is that long time-series of population change are seldom available - a typical time series for a forest insect population has observations for $10 \mathrm{~s}$ of years - while the fitting methods require very long time series - 10 s of 1000 s of observations are needed to reliably fit chaotic time series (Turchin and Taylor, 1992).

The power of the interaction function, $\phi$, is that it allows prediction of population dynamics through local observations of resource sites on a single generation without the analysis of long and difficult to obtain time series. We first describe the competition of individuals in terms of usage of discrete resource sites over which, on each generation, the individuals are distributed at random. We then write down the appropriate interaction function for such competition and thus derive the equivalent difference equation model. This approach works even when the population dynamics are periodic or chaotic.

Sumpter and Broomhead (2001) applied exactly this approach in predicting the population levels of a parasitic mite, Varroa in the Asian honey bee, which exhibited scramble competition. Data to parameterise interaction functions has also been collected, for example, for two species of bean bruchids: Callosobruchus analis which larvae exhibit contest competition and C. phaseoli which larvae exhibit scramble competition (Toquenaga and Fujii, 1991). Indeed, a whole range of competition and interference patterns have been observed between these beetles (Takano et al., 2001). Coupled with information about how the beetles distribute themselves between beans and data of fecundity, very strong predictions about the dynamics of laboratory populations should be possible.

The link between population dynamics and interaction function goes both ways. Where it is impossible to collect the data required to parameterise an interaction function, population dynamics may be used to infer that interaction function. For example, time series data may be used to determine whether a particular species has a scramble or contest competition, or to determine aspects of predator-prey local interactions. Some population dynamic models, such as the logistic map, have no biologically realistic interaction function, suggesting either that they are poor models or not explainable in terms of non-overlapping generations with random distribution of individuals at discrete interaction sites.

While the density approximation relates interaction function to mean population dynamics, the normal approximation gives a relationship between the mean of an interaction function and its variance, assuming both are derived from a particular interaction function. This relationship has never, to our knowledge, been accounted for when fitting models to ecological time series. For example, fitting the Ricker map to noisy data involves fitting either

$\rho x_{t} e^{-x_{t}}+\sigma \varepsilon_{t} \quad$ or $\rho x_{t} e^{-x_{t}} e^{\sigma \varepsilon_{t}}$, 
where $\varepsilon_{t} \sim N(0,1)$ (Turchin and Taylor, 1992; Berryman, 1999). However, when fitting to site-based models at least, $\sigma$ should be considered a function of $x_{t}$ and is not independent of $\rho$. Indeed, the proper way to fit the Ricker map to data generated by a well-mixed site-based system is by fitting the independent parameters $\rho$ and $n$ in the stochastic difference equation

$\rho x_{t} e^{-x_{t}}+\sqrt{\frac{v\left(x_{t}\right)}{n}} \varepsilon_{t}$,

where $v$ is the noise term from Eq. (25). Caution is required here, however, since Eq. (40) captures demographic noise only, i.e. stochasticity resulting from interactions amongst individuals. In real ecological time series, there is also variation in the environment, modelled by appropriate changes in $\rho$ and $n$. Determining the relative importance of demographic and environmental noise and fitting appropriate models to ecological data will require further theoretical development.

\subsection{Spatial scale}

A central assumption made in describing an ecological system as a well-mixed site-based model, is that all interactions take place at discrete sites. While this may hold for many ecological systems, such as those described in the introduction, there are many more systems for which this assumption fails to hold: individuals often disperse globally but compete over a continuous resource environment. We can however think of the total continuous resource area of size $a$ being split into smaller resource areas of size $r$, such that $n=a / r$ is the number of resource units. $r$ corresponds to a range over which an individual competes with other individuals. If we are then to fit, for example, Eq. (40) to data where individuals compete over a continuous and stable resource environment the value of $n$ obtained should be approximately equal to $a / r$. This observation could be used, in the absence of environmental noise, to determine species interaction ranges from time series data.

When two or more species interact they often do so at two very different spatial scales. For example, an area containing thousands of insects may contain zero or one predatory bird (Gurney and Nisbet, 1998). This disparity of scales can be incorporated into our models by first identifying the scale for each of the species, then distributing fractions of the species amongst resource sites. For example, if there are $y_{t}$ birds, we could distribute $r y_{t}$ bird attack units and $x_{t}$ insects amongst the $n$ resource units. In this case, $r$ would be the maximum number of resource units that a bird could search for insects. A sensible interaction function could thus be constructed and density approximation made, though it is questionable as to whether the normal approximation will also apply. Indeed, the variance may depend on the shape of the search area for the birds. Such models require more thorough investigation to establish their biological realism and are a ripe area for future research.

\subsection{Local dispersal}

Another assumption made about the site-based models we discuss in this paper is that dispersal is global. Although interactions take place at local resource sites, between generations individuals are well-mixed over the entire system, i.e. they can move to any of the $n$ sites. In many of the individual-based models applied in understanding ecological systems dispersal is local, and much of the interesting dynamics observed in these models is due to the resulting spatial effects (Diekmann et al., 2000). One of the intriguing questions regarding these systems is how to capture their behaviour of a complicated explicitly spatial system in a small set of equations. To address this problem, Rand and Wilson (1995) used delay embedding techniques to show that the dynamics of a spatial resource-predatorprey model could be captured in a set of four differential equations. Pascual and Levin (1999) describe a spatial predator-prey model, not far removed in terms of interactions from the predator-prey model we discuss above, and show when sampling of the population is made at the appropriate spatial scale the dynamics are well approximated by two differential equations. Such observations are encouraging since they indicate that, on some spatial scale at least, the population dynamics of spatial site-based models can be approximately equivalent to those of a small number of differential equations.

The important question then is how to relate an interaction function and a set of rules for local dispersal to a simple dynamical system consisting of a handful of equations, without resorting to statistical fitting techniques. The well-mixed site models discussed in this paper show how this relationship is established when the distances moved between interactions is large. Looking at this relationship is in itself useful since it separates the effects of discreteness and stochasticity in interactions from those induced by the fact that interactions are local in space. It is moreover possible that by identifying the dispersal scale associated with a particular spatial model, we may be able to approximate local dynamics at that scale using a local normal approximation. Indeed, the normal approximation was seen to apply to systems where $n$ was as small as 100 (contest competition) or 200 (predator-prey model), while the characteristic dispersal scale identified by Pascual and Levin (1999) was as large as $n=50 \times 50=2500$. Approximation of such a spatial model would thus take the form of a lattice of stochastic coupled difference equations. Mathematical analysis of these coupled 
equations would be considerably easier than analysis of the original spatially explicit site-based model. Indeed, coupled map lattice approximations may prove a useful alternative to the higher-order moment approximation techniques (Diekmann et al., 2000).

\subsection{Evolutionary dynamics}

The example of contest vs. scramble competition which we analyse using the interaction function in Eq. (27) is somewhat contrived. We may equally well see contest individuals always beating scramble individuals at the resource sites (see, for example, Toquenaga and Fujii, 1990b). We use the example to illustrate the connection between our approach and the dynamical systems approach to evolutionary game theory (Hofbauer and Sigmund, 1998): our interaction function, $\phi$, can be viewed as a payoff function and $\Phi$ as a replicator equation. In the dynamical systems approach to game theory the replicator equations take the form of Lotka-Voltera equations, the equilibrium solution of which determine the evolutionary stable strategy. The same logic applies here, the solution to $\Phi$ gives the evolutionary stable strategy for $\phi$. The added feature in our case is the more general and realistic nature of the dynamics described by $\Phi$, which are obtained by the local interactions between individuals. Different strategies produce not only different payoffs but also different population dynamics. In the example of contest vs. scramble competition, the evolutionary stable strategy of scramble produced chaotic dynamics and led to a 'tragedy of the commons' whereby a resource was under exploited by the group as a whole.

In comparing contest and scramble reproduction both in terms of population dynamics and in terms of evolutionary stable strategies we have given a clear example of the relationship between population dynamics and evolution. In many ecological systems, population dynamics and evolution run on similar time-scales and both play a role in determining the overall population dynamics. If we extend the contest vs. scramble model so that movement between generations is not well-mixed (i.e. using a local dispersal model) we see co-existence between scramble and contest competition despite the fact that scramble always defeats contest at a local resource site and both have equal fecundity. This observation can be explained by the locally unstable dynamics of scramble competition compared to the always stable dynamics of contest competition. The coupling of population dynamics and evolution in this way thus provides some insight into the age-old problem of species co-existence. We hope that the results presented in this paper will prove useful for analysing other questions of this type.

\section{Acknowledgments}

We thank Minus van Baalen, Åke Brännström, Laurent Lehmann, Max Reuter, Jaroslav Stark and especially David Broomhead for discussions contributing to this work. We also thank the two anonymous referees for their detailed reading and corrections of this paper. This research was funded by EPSRC (UK) and STINT (Sweden).

\section{Appendix A. Proofs}

Proof (of Proposition 1). The conditional expectation of the population at the next generation, $\mathbf{M}_{t+1}$ given that the current population $\mathbf{M}_{t}=\mathbf{m}=n \mathbf{X}_{t}$, is the sum of the expected number of offspring produced at each site. Thus,

$$
\begin{aligned}
E\left[\mathbf{M}_{t+1} \mid \mathbf{M}_{t}=\mathbf{m}\right]= & \sum_{i=1}^{n} E\left[\phi\left(\mathrm{C}_{i}, \xi_{i}\right) \mid \mathbf{M}_{t}=\mathbf{m}\right] \\
= & \sum_{i=1}^{n} \sum_{\mathbf{k} \in \mathbb{Z}^{s}} \operatorname{Pr}\left\{\mathrm{C}_{i}=\mathbf{k} \mid \mathbf{M}_{t}=\mathbf{m}\right\} \\
& \times E\left[\phi\left(\mathbf{k}, \xi_{i}\right) \mid \mathrm{C}_{i}=\mathbf{k}\right] .
\end{aligned}
$$

Since the animals are distributed uniformly at random between $n$ cells, the probability that a particular site contains a particular individual of species $j$ is $1 / n$. Thus, the probability there are $k_{j}$ individuals of species $j$ at a particular site is

$$
\left(\begin{array}{c}
m_{j} \\
k_{j}
\end{array}\right)(1 / n)^{k_{j}}(1-1 / n)^{m_{j}-k_{j}}
$$

where $k_{j}$ is the $j$ th element of $\mathbf{k}$. Since the distribution of each species is independent

$\operatorname{Pr}\left\{\mathbf{C}_{i}=\mathbf{k} \mid \mathbf{M}_{t}=\mathbf{m}\right\}=\left(\begin{array}{c}\mathbf{m} \\ \mathbf{k}\end{array}\right)(1 / n)^{\mathbf{k}}(1-1 / n)^{\mathbf{m}-\mathbf{k}}$,

where for notational convenience,

$$
\left(\begin{array}{c}
\mathbf{m} \\
\mathbf{k}
\end{array}\right)=\prod_{j=1}^{s}\left(\begin{array}{c}
m_{j} \\
k_{j}
\end{array}\right) \text { and } a^{\mathbf{k}}=\prod_{j=1}^{s} a^{k_{j}} .
$$

Eq. (A.1) thus becomes

$$
\begin{aligned}
E\left[\mathbf{M}_{t+1} \mid \mathbf{M}_{t}=\mathbf{m}\right]= & \sum_{i=1}^{n} \sum_{\mathbf{k} \in \mathbb{Z}^{s}}\left(\begin{array}{c}
\mathbf{m} \\
\mathbf{k}
\end{array}\right)(1 / n)^{\mathbf{k}}(1-1 / n)^{\mathbf{m}-\mathbf{k}} \\
& \times E\left[\phi\left(\mathbf{k}, \xi_{i}\right)\right] .
\end{aligned}
$$

Since the distribution of $\xi_{i}$ is independent of the site for all $i$,

$$
\begin{aligned}
E\left[\mathbf{M}_{t+1} \mid \mathbf{M}_{t}=\mathbf{m}\right]= & n \sum_{\mathbf{k} \in \mathbb{Z}^{s}}\left(\begin{array}{c}
\mathbf{m} \\
\mathbf{k}
\end{array}\right)(1 / n)^{\mathbf{k}}(1-1 / n)^{\mathbf{m}-\mathbf{k}} \\
& \times E\left[\phi\left(\mathbf{k}, \xi_{i}\right)\right] .
\end{aligned}
$$


Recall that the population density is $\mathbf{X}_{t}=\mathbf{M}_{t} / n$. Proposition 1 then follows from the observation that for large $n, n^{k} \approx n ! /(n-k) !$ and

$$
\left(\begin{array}{c}
\mathbf{m} \\
\mathbf{k}
\end{array}\right)(1 / n)^{\mathbf{k}}(1-1 / n)^{\mathbf{m}-\mathbf{k}} \approx \frac{\mathbf{x}^{\mathbf{k}}}{\mathbf{k} !}(1-1 / n)^{\mathbf{m}-\mathbf{k}} \rightarrow e^{-\mathbf{x}} \frac{\mathbf{x}^{\mathbf{k}}}{\mathbf{k} !}
$$

as $n \rightarrow \infty$. Substituting the right-hand side into Eq. (A.2) for each $\mathbf{k}$ gives

$$
E\left[\mathbf{X}_{t+1} \mid \mathbf{X}_{t}=\mathbf{x}\right] \rightarrow \Phi(\mathbf{x}):=e^{-\mathbf{x}} \sum_{\mathbf{k} \in \mathbb{Z}^{s}} \frac{\mathbf{x}^{\mathbf{k}}}{\mathbf{k} !} E\left[\phi\left(\mathbf{k}, \xi_{i}\right)\right]
$$

as $n \rightarrow \infty$, as in Proposition 1 .

Proof (of Theorem 2). The proof is based on the observation that for large $n$ the distribution of the site count $\mathrm{C}=\left(\mathrm{C}_{1}, \ldots, \mathrm{C}_{n}\right)$ can be derived from (though is not equal to) the distribution of $n$ independent random variables $\mathrm{U}=\left(\mathrm{U}_{1}, \ldots, \mathrm{U}_{n}\right)$ with identical distribution, $\cup_{i} \sim \operatorname{Po}(\mathbf{x})$ (s-dimensional Poisson distribution), where $\mathbf{M}_{t}=\mathbf{m}=n \mathbf{x}$ is the number of individuals on generation $t$ (Kolchin et al., 1978). Step 1 is to show that conditioned on $\sum_{i} \mathrm{U}_{i}=\mathbf{m}$ the distributions of $\mathbf{C}$ and $U$ are equal. In step 2, we define a class of random variables with elements

$\mathrm{D}_{\phi}:=\frac{1}{\sqrt{n}}\left(\sum_{i=1}^{n} \phi\left(\mathrm{U}_{i}, \xi_{i}\right)-n \Phi(\mathbf{x})\right)$,

where $\phi$ is the interaction function. By the central limit theorem, as $n \rightarrow \infty, \mathrm{D}_{\phi}$ has mean 0 and a known variance. The question is then to relate the variance of $\mathrm{D}_{\phi}$ to the variance of Eq. (6) (for the $C_{i}$ 's). We begin this, in step 3, by first constructing a family of interaction functions with the property that when $\sum_{i} \mathrm{\cup}_{i}=\mathbf{m}, \mathrm{D}_{K_{j}}=0$, for $j=1, \ldots, s$. In step 4 , we construct a $\mathrm{D}_{\zeta}$ which is uncorrelated with, and thus converges to a random variable independent of, the $\mathrm{D}_{K_{j}}$ 's. Through this independence, the limit distribution of Eq. (6) is seen to be equivalent to the limit distribution of $D_{\zeta}$. The variance in Eq. (7), which is equal to the variance of the limit distribution of $\mathbf{D}_{\zeta}$, is thus obtained.

Step 1. We start by showing that the uniform distribution of $\mathrm{C}=\left(\mathrm{C}_{1}, \ldots, \mathrm{C}_{n}\right)$ is equal to that of $\mathrm{U}=\left(\mathrm{U}_{1}, \ldots, \mathrm{U}_{n}\right)$ where each element has identical distribution $\cup_{i} \sim \operatorname{Po}(\mathbf{x})$, conditioned on the event $\sum_{i} \mathrm{U}_{i}=\mathbf{m}=n \mathbf{x}$. The distribution of $\mathrm{C}$ can be expressed as follows. For each of the $\mathbf{m}$ individuals, we choose uniformly at random and independently one of $n$ sites to put that specific individual into. The distribution across all sites is then multinomial.
That is

$$
\begin{aligned}
\operatorname{Pr} & \left\{\mathrm{C}_{1}=\mathbf{k}_{1}, \ldots, \mathrm{C}_{n}=\mathbf{k}_{n}\right\} \\
& =\left(\begin{array}{c}
\mathbf{m} \\
\mathbf{k}_{1}, \ldots, \mathbf{k}_{n}
\end{array}\right) / n^{\mathbf{m}} \\
& =\frac{\mathbf{m} !}{n^{\mathbf{m}}} \prod_{i=1}^{n} \frac{1}{\mathbf{k}_{i} !} \\
& =\mathbf{m} ! \frac{e^{-\mathbf{m}^{\mathbf{m}} \mathbf{\mathbf { m }}}}{e^{-\mathbf{m}_{\mathbf{m}}^{\mathbf{m}}}} \prod_{i=1}^{n} \frac{1}{\mathbf{k}_{i} !} \\
& =\frac{e^{-\mathbf{m} \mathbf{x}^{\mathbf{m}}}}{\operatorname{Pr}\left\{\sum_{i} \mathrm{U}_{i}=\mathbf{m}\right\}} \prod_{i=1}^{n} \frac{1}{\mathbf{k}_{i} !} \\
& =\frac{\operatorname{Pr}\left\{\mathrm{U}_{1}=\mathbf{k}_{1}, \ldots, \mathrm{U}_{n}=\mathbf{k}_{n}\right\}}{\operatorname{Pr}\left\{\sum_{i=1}^{n} \mathrm{U}_{i}=\mathbf{m}\right\}} \\
& =\operatorname{Pr}\left\{\mathrm{U}_{1}=\mathbf{k}_{1}, \ldots, \mathrm{\cup}_{n}=\mathbf{k}_{n} \mid \sum_{i=1}^{n} \mathrm{U}_{i}=\mathbf{m}\right\} .
\end{aligned}
$$

Thus, the distributions of $\mathrm{C}$ and $\mathrm{U}$ conditioned on $\sum_{i} \mathrm{U}_{i}=\mathbf{m}$ are equal.

Step 2. Consider the class $\mathscr{D}$ of random variables $\mathrm{D}_{\phi}$

$\mathrm{D}_{\phi}:=\frac{1}{\sqrt{n}}\left(\sum_{i=1}^{n} \phi\left(\mathrm{U}_{i}, \xi_{i}\right)-n \Phi(\mathbf{x})\right)$,

where $\Phi(\mathbf{x})$ is the density approximation given by Eq. (3). Since the $U_{i}$ are independent identically distributed random variables, by the central limit theorem, an element of $\mathscr{D}, \mathrm{D}_{\phi}$ converges in distribution to a Gaussian random variable as $n \rightarrow \infty$ with $\mathbf{x}$ fixed. Moreover, by the 'multidimensional' central limit theorem, the joint limit distribution of $\mathrm{D}_{\phi}=\left(\mathrm{D}_{\phi_{1}}, \ldots, \mathrm{D}_{\phi_{s}}\right)$ is an $s$-dimensional Gaussian distribution with mean $\mathbf{0}$ and variance matrix

$$
\begin{aligned}
\operatorname{Var}\left[\mathrm{D}_{\mathrm{U}, \phi}\right]= & \operatorname{Var}[\phi] \\
:= & e^{-\mathbf{x}} \sum_{\mathbf{k} \in \mathbb{Z}^{s}} \frac{\mathbf{x}^{\mathbf{k}}}{\mathbf{k} !} E\left[\phi\left(\mathbf{k}, \xi_{i}\right) \phi\left(\mathbf{k}, \xi_{i}\right)^{\mathrm{T}}\right] \\
& -\Phi(\mathbf{x}) \Phi(\mathbf{x})^{\mathrm{T}} .
\end{aligned}
$$

We can consider the correlation between two elements of $\mathscr{D}$ using

$$
\begin{aligned}
\operatorname{Cov}\left[\mathrm{D}_{\phi_{1}}, \mathrm{D}_{\phi_{2}}\right]= & e^{-\mathbf{x}} \sum_{\mathbf{k}} \frac{\mathbf{x}^{\mathbf{k}}}{\mathbf{k} !} E\left[\phi_{1}\left(\mathbf{k}, \xi_{i}\right) \phi_{2}\left(\mathbf{k}, \xi_{i}\right)^{\mathrm{T}}\right] \\
& -\Phi_{1}(\mathbf{x}) \Phi_{2}(\mathbf{x})^{\mathrm{T}} .
\end{aligned}
$$

Uncorrelated elements will have $\operatorname{Cov}\left[\mathrm{D}_{\phi_{1}}, \mathrm{D}_{\phi_{2}}\right]=0$. Furthermore, in the limit $n \rightarrow \infty$, when all elements are normally distributed, the condition that $\operatorname{Cov}\left[\mathrm{D}_{\phi_{1}}, \mathrm{D}_{\phi_{2}}\right]=$ 0 implies that $D_{\phi_{1}}$ and $D_{\phi_{2}}$ are independent (see, for example, Capinski and Kopp, 1999, Chapter 6). Note that $\mathscr{D}$ is a Hilbert space with an inner product defined by the covariance in Eq. (A.5).

Step 3. Let $\mathrm{D}_{K_{j}}, j=1, \ldots, s$ be the elements of $\mathscr{D}$ given by the interaction functions defined by

$K_{j}(\mathbf{k}, u)=k_{j} \mathbf{1}_{s}$, 
i.e. $K_{j}$ is the interaction function for which the number of individuals produced of each species is equal to $k_{j}$, the number of species $j$ at the site (this is only to be considered as a mathematical construct, and not as anything biologically realistic!). Note that

$\mathrm{D}_{K_{j}}=\frac{1}{n}\left(\sum_{i=1}^{n} k_{j}-n x_{j}\right)$,

which is equal to zero, under the condition used in step 1 that $\sum_{i=1}^{n} \mathrm{U}_{i}=n \mathbf{x}$ and $\mathrm{D}_{K_{j}}=0$.

By Eq. (A.5), for $\mathrm{D}_{\phi} \in \mathscr{D}$,

$$
\begin{aligned}
\operatorname{Cov}\left[\mathrm{D}_{\phi}, \mathrm{D}_{K_{j}}\right] & =e^{-\mathbf{x}} \sum_{\mathbf{k}} \frac{k_{j} \mathbf{x}^{\mathbf{k}}}{\mathbf{k} !} \phi(\mathbf{k})-x_{j} \Phi[\phi](\mathbf{x}) \\
& =x_{j}\left(e^{-\mathbf{x}} \sum_{\mathbf{k}} \frac{k_{j} \mathbf{x}^{\mathbf{k}}}{x_{j} \mathbf{k} !} \phi(\mathbf{k})-\Phi(\mathbf{x})\right) \\
& =x_{j} \frac{\partial \Phi}{\partial x_{j}} .
\end{aligned}
$$

Note that the $\mathrm{D}_{K_{j}}, j=1, \ldots, s$ form an orthogonal set, in that $\operatorname{Cov}\left[\mathrm{D}_{K_{i}}, \mathrm{D}_{K_{j}}\right]=0$ if $i \neq j$. Furthermore, $\operatorname{Var}\left[\mathrm{D}_{K_{j}}\right]=\operatorname{Cov}\left[\mathrm{D}_{K_{j}}, \mathrm{D}_{K_{j}}\right]=x_{j}$.

Step 4. Given an interaction function $\phi$, we can construct the $\mathrm{D}_{\zeta_{l}}, l=1, \ldots, s$, which are the orthogonal projection of each $D_{\phi_{l}}$ onto the subspace spanned by $\mathrm{D}_{K}=\left(\mathrm{D}_{K_{1}}, \ldots, \mathrm{D}_{K_{s}}\right)$. These are given by

$$
\begin{aligned}
\mathrm{D}_{\zeta_{l}}= & \mathrm{D}_{\phi_{l}}-\frac{1}{x_{1}} \operatorname{Cov}\left[\mathrm{D}_{\phi_{l}}, \mathrm{D}_{K_{1}}\right] \mathrm{D}_{K_{1}}-\cdots \\
& -\frac{1}{x_{s}} \operatorname{Cov}\left[\mathrm{D}_{\phi_{l}}, \mathrm{D}_{K_{s}}\right] \mathrm{D}_{K_{s}} \\
= & \mathrm{D}_{\phi_{l}}-\sum_{j=1}^{s} \frac{\partial \Phi_{l}}{\partial x_{j}} \mathrm{D}_{K_{j}}
\end{aligned}
$$

by Eq. (48). Note that

$$
\begin{aligned}
\operatorname{Cov}\left[\mathrm{D}_{\zeta_{l}}, \mathrm{D}_{K_{i}}\right] & =\operatorname{Cov}\left[\mathrm{D}_{\phi_{l}}, \mathrm{D}_{K_{i}}\right]-\sum_{j=1}^{s} \frac{\partial \Phi_{l}}{\partial x_{j}} \operatorname{Cov}\left[\mathrm{D}_{K_{j}}, \mathrm{D}_{K_{i}}\right] \\
& =0
\end{aligned}
$$

for all $i$, so $\mathrm{D}_{\zeta_{l}}$ is indeed orthogonal to all $\mathrm{D}_{K_{i}}, i=$ $1, \ldots, s$. Thus, with $\mathrm{D}_{\zeta}=\left(\mathrm{D}_{\zeta_{1}}, \ldots, \mathrm{D}_{\zeta_{s}}\right)$, we have

$\mathrm{D}_{\phi}=\mathrm{D}_{\zeta}+\frac{\partial \Phi}{\partial \mathbf{x}} \mathrm{D}_{K}$

where $\frac{\partial \Phi}{\partial \mathbf{x}}$ is the Jacobian matrix of partial derivatives of $\Phi$. Since each $\mathrm{D}_{\zeta_{1}}$ is orthogonal to all $\mathrm{D}_{K_{j}}, \mathrm{D}_{\zeta}$ converges to a Gaussian random variable that is independent of the Gaussian limit distributions of $\mathrm{D}_{K_{j}}, j=1, \ldots, s$. Thus as $n \rightarrow \infty$, by the multivariate central limit theorem, $\mathbf{D}_{\zeta}$ has variance

$\operatorname{Var}\left[\mathrm{D}_{\zeta}\right]=\operatorname{Var}\left[\mathrm{D}_{\phi}\right]-\frac{\partial \Phi}{\partial \mathbf{x}} \operatorname{diag} \mathbf{x} \frac{\partial \Phi^{\mathrm{T}}}{\partial \mathbf{x}}$,

where $\operatorname{diag} \mathbf{x}$ is the diagonal matrix having $\mathbf{x}=$ $\left(x^{1}, \ldots, x^{s}\right)$ along the diagonal.
To conclude the proof, it remains to show that the variance given in Eq. (A.10) is indeed the variance given in the statement of the theorem. To show this we note from step 1 that the distribution of $C$ equals that of $U$ given that $\sum_{i=1}^{n} \bigcup_{i}=n \mathbf{x}$. Thus under this condition, by Eq. (A.9)

$\frac{1}{\sqrt{n}}\left(\sum_{i=1}^{n} \phi\left(\mathrm{C}_{i}, \xi_{i}\right)-n \Phi(\mathbf{x})\right)$

is equal in distribution to $D_{\phi}$. But we showed in step 3 that when $\sum_{i=1}^{n} \mathrm{U}_{i}=n \mathbf{x}, \mathrm{D}_{K_{j}}=0$, so under this condition Eq. (A.11) is also equal in distribution to $D_{\phi}-$ $\frac{\partial \Phi}{\partial \mathbf{x}} \mathbf{D}_{\mathbf{k}}$. Moreover, since $\mathbf{D}_{\zeta}$, in the limit, is stochastically independent of $\mathrm{D}_{K_{i}}$, it follows that the limit distribution of Eq. (A.11) equals the limit distribution of $\mathrm{D}_{\zeta}$. And thus, by Eq. (A.10), the limit distribution of Eq. (6) is

$v(\mathbf{x})=\operatorname{Var}\left[\mathbf{D}_{\zeta}\right]=\operatorname{Var}[\phi]-\frac{\partial \Phi}{\partial \mathbf{x}} \operatorname{diag} \mathbf{x} \frac{\partial \Phi^{\mathrm{T}}}{\partial \mathbf{x}}$

as $n \rightarrow \infty$ and as stated in the theorem.

Proof (of Corollary 3). (corollary and proof were made by D.S. Broomhead). The proof involves iterating and repeatedly linearising Eq. (9):

$$
\begin{aligned}
\mathbf{x}_{t+1}= & \Phi\left(\mathbf{x}_{t}\right)+\frac{\varepsilon_{t}\left(\mathbf{x}_{t}\right)}{\sqrt{n}} \\
= & \Phi\left(\Phi\left(\mathbf{x}_{t-1}\right)+\frac{\varepsilon_{t-1}\left(\mathbf{x}_{t-1}\right)}{\sqrt{n}}\right)+\frac{\varepsilon_{t}\left(\mathbf{x}_{t}\right)}{\sqrt{n}} \\
\approx & \Phi\left(\Phi\left(\mathbf{x}_{t-1}\right)\right)+\mathbf{J}\left(\Phi\left(\mathbf{x}_{t-1}\right)\right) \frac{\varepsilon_{t-1}\left(\mathbf{x}_{t-1}\right)}{\sqrt{n}}+\frac{\varepsilon_{t}\left(\mathbf{x}_{t}\right)}{\sqrt{n}} \\
\approx & \Phi\left(\Phi\left(\Phi\left(\mathbf{x}_{t-2}\right)\right)\right)+\mathbf{J}\left(\Phi\left(\Phi\left(\mathbf{x}_{t-2}\right)\right)\right) \mathbf{J}\left(\Phi\left(\mathbf{x}_{t-2}\right)\right) \\
& \times \frac{\varepsilon_{t-2}\left(\mathbf{x}_{t-2}\right)}{\sqrt{n}}+\mathbf{J}\left(\Phi\left(\mathbf{x}_{t-1}\right)\right) \frac{\varepsilon_{t-1}\left(\mathbf{x}_{t-1}\right)}{\sqrt{n}}+\frac{\varepsilon_{t}\left(\mathbf{x}_{t}\right)}{\sqrt{n}} \\
\approx & \ldots \ldots,
\end{aligned}
$$

where $\mathbf{J}\left(\mathbf{x}_{t}\right)=\left.\frac{\partial \Phi}{\partial \mathbf{x}}\right|_{\mathbf{x}=\mathbf{x}_{t}}$ is the Jacobian. When the iterations of Eq. (9) occur round a stable fixed point, $\mathbf{x}_{t} \approx \Phi\left(\mathbf{x}_{t}\right) \approx \mathbf{x}_{t+1} \approx \mathbf{x}_{*}$ for all $t$ so that

$\mathbf{x}_{t+1} \approx \mathbf{x}_{*}+\sum_{\tau=0}^{t} \mathbf{J}\left(\mathbf{x}_{*}\right)^{\tau} \frac{\varepsilon_{t-\tau}\left(\mathbf{x}_{*}\right)}{\sqrt{n}}$.

$\mathbf{x}_{t+1}$ is thus normally distributed with mean $\mathbf{x}_{*}$ and variance $w\left(\mathbf{x}_{*}\right) / n$, where

$w\left(\mathbf{x}_{*}\right):=\operatorname{Var}\left[\mathbf{X}_{t} \mid X_{0}=x_{*}\right] \approx \sum_{\tau=0}^{\infty} \mathbf{J}\left(\mathbf{x}_{*}\right)^{\tau} v\left(\mathbf{x}_{*}\right) \mathbf{J}\left(\mathbf{x}_{*}\right)^{\tau^{\mathrm{T}}}$

as given in Eq. (10).

Proof (of Theorem 4). We do not provide a full proof of Cramérs theorem (see, for example, the text book of Dembo and Zeitouni, 1992). However, we note that Cramérs theorem, like the central limit theorem, is seen to hold for any random variable which is the mean of 
$n$ independent random variables. From its definition, in Eq. (2), $\mathbf{X}_{t+1}$, is a sum of random variables, though these are not independent. We thus rely on the fact that as $n \rightarrow \infty, \quad \mathbf{X}_{t+1}$ is well approximated by a sum of independent Poisson distributed random variables (as in the proof of Theorem 2). We note however that in Eq. (12) we do not define the log generating function of some adjusted function $\zeta$ but instead that of $\phi$. This replacement leads to the $o(1)$ term in the exponential on the right-hand sides of the equation in Theorem 4 and of Eq. (15).

\section{References}

Berryman, A.A., 1999. Principles of Population Dynamics and their Application. Stanley Thornes (Publishers), Cheltenham, UK, pp. 243.

Boerlijst, M., Lamers, M.E, Hogeweg, P., 1993. Evolutionary consequences of spiral waves in a host-parasitoid system. Proc. R. Soc. London Ser. B 253, 15-18.

Bolker, B., Grenfell, B., 1995. Space, persistence and dynamics of measles epidemics. Philos. Trans. R. Soc. London Ser. B 348, 309-320.

Capinski, M., Kopp, E., 1999. Measure, Integral and Probability. Springer, London, pp. 227.

Czaran, T., 1998. Spatiotemporal Models of Population and Community Dynamics. Chapman \& Hall, London.

DeAngelis, D.L., Gross, L.J., 1992. Individual-based Models and Approaches in Ecology: Populations, Communities and Ecosystems. Chapman \& Hall, London.

Dembo, A., Zeitouni, O., 1992. Large Deviation Techniques. Jones and Bartlett Publishers, London, pp. 346.

Diekmann, U., Law, R., 2000. Relaxation projections and the method of moments. In: Diekmann, U., et al. (Ed.), The Geometry of Ecological Interactions. Cambridge University Press, Cambridge, pp. 412-455.

Diekmann, U., Law, R., Metz, J.A.J., 2000. The Geometry of Ecological Interactions. Cambridge University Press, Cambridge, pp. 565.

Durrett, R., Levin, S.A., 1994a. Stochastic spatial models: a users' guide to ecological applications. Philos. Trans. R. Soc. London Ser. B 343, 329-350.

Durrett, R., Levin, S.A., 1994b. The importance of being discrete and spatial. Theor. Popul. Biol. 46, 363-395.

Ermentrout, G., Edelstein-Keshet, L., 1992. Cellular automata approaches to biological modelling. J. Theor. Biol. 160, 97-133.

Gurney, W.S.C., Nisbet, R.M., 1998. Ecological Dynamics. Oxford University Press, Oxford, pp. 335.

Hanski, I., 1999. Metapopulation Ecology. Oxford University Press, Oxford, pp. 313

Hofbauer, J., Sigmund, K., 1998. Evolutionary Games and Population Dynamics. Cambridge University Press, Cambridge, pp. 323.

Iwao, K., Ohsaki, N., 1996. Inter- and intraspecific interactions among larvae of specialist and generalist parasitoids. Resource Popul. Ecol. 38, 265-273.

Iwasa, Y., Nakamaru, M., Levin, S.A., 1998. Allelopathy of bacteria in a lattice population: competition between colicin-sensitive and colicin-producing strains. Evol. Ecol. 12, 785-802.

Keeling, M.J., 2002. Using individual-based simulations to test the Levins metapopulation paradigm. J. Anim. Ecol. 71, 270-279.
Keeling, M.J., Gilligan, C.A., 2000. Metapopulation dynamics of bubonic plague. Nature 407, 903-906.

Keeling, M.J., Wilson, H.B., Pacala, S.W., 2000. Reinterpreting space, time lags, and functional responses in ecological models. Science 290, 1758-1761.

Keeling, M.J., Wilson, H.B., Pacala, S.W., 2002. Deterministic limits to stochastic spatial models of natural enemies. Am. Nat. 159, 57-80.

Keeling, M.J., et al., 2001. Dynamics of the 2001 UK foot and mouth epidemic: stochastic dispersal in a heterogeneous landscape. Science 294, 813-817.

Kerr, B., Riley, M.A., Feldman, M.W., Bohannan, B.J.M., 2002. Local dispersal promotes biodiversity in a real-life game of rockpaper-scissors. Nature 418, 171-174.

Kolchin, V.F., Sevastyanov, B.A., Chistyakov, V.P., 1978. Random Allocations. Winston-Wiley, New York.

Levins, R., 1969. Some demographic and genetic consequences of environmental heterogeneity for biological control. Bull. Entomol. Soc. Am. 15, 237-240.

Levins, R., 1970. Extinction. Lecture Notes Math. 2, 75-107.

Nicholson, A.J., 1954. An outline of the dynamics of animal populations. Aust. J. Zool. 2, 9-65.

Nowak, M.A., May, R.M., 1992. Evolutionary games and spatial chaos. Nature 359, 826-829.

Pacala, S.W., 1986a. Neighbourhood models of plant population dynamics. 2. Multispecies models of annuals. Theor. Popul. Biol. 29, 262-292.

Pacala, S.W., 1986b. Neighbourhood models of plant population dynamics. 4. Single-species and multispecies models of annuals with dormant seeds. Am. Nat. 128, 859-878.

Pacala, S.W., Silander, J.A., 1985. Neighbourhood models of plant population dynamics. 1. Single-species models of annuals. Am. Nat. 125, 385-411.

Pacala, S.W., Tilman, D., 1996. Limiting similarity in mechanistic and spatial models of plant competition in heterogeneous environments. Am. Nat. 143, 222-257.

Pascual, M., Levin, S.A., 1999. From individuals to population densities: searching for the intermediate scale of nontrivial determinism. Ecology 80, 2225-2236.

Prinkkila, M-L., Hanski, I., 1995. Complex competitive interactions in four species of Lucilia blowflies. Ecol. Entomol. 20, 261-272.

Rand, D.A., 1999. Correlation equations and pair approximations for spatial ecologies. In: McGlade, J. (Ed.), Theoretical Ecology, Vol. 2, Blackwell, Oxford, pp. 140-142.

Rand, D.A., Wilson, H.B., 1995. Using spatio-temporal chaos and intermediate-scale determinism to quantify spatially extended ecosystems. Proc. R. Soc. London Ser. B 259, 111-117.

Rath, W., 1999. Co-adaptation of Apis cerana Fabr. and Varroa jacobsoni Oud. Apidologie 30, 97-110.

Royama, T., 1992. Analytical Population Dynamics. Chapman \& Hall, London, pp. 369.

Sumpter, D.J.T., Broomhead, D.S., 2001. Relating individual behaviour to population dynamics. Proc. R. Soc. London Ser. B 268, 925-932.

Takano, M., Toquenaga, Y., Fujii, K., 2001. Polymorphism of competition type and its genetics in Callosobruchus maculatus (Coleoptra: Bruchidae). Popul. Ecol. 43, 265-273.

Thomas, J.A., Elmes, G.W., Wardlaw, J.C., 1993. Contest competition among Maculinea rebeli butterfly larvae in ant nests. Ecol. Entomol. 18, 73-76.

Toquenaga, Y., 1993. Contest and scramble competitions in Callosobruchus maculatus (Coleoptra: Bruchidae) II. Larval competition and interference mechanisms. Resource Popul. Ecol. 35, $57-68$.

Toquenaga, Y., Fujii, K., 1990a. Contest and scramble competition between two bruchid species (Coleoptra: Bruchidae) I. Larval 
competition curves and interference mechanisms. Resource Popul. Ecol. 32, 349-363.

Toquenaga, Y., Fujii, K., 1990b. Contest and scramble competition between two bruchid species (Coleoptra: Bruchidae) III. Multiplegeneration competition experiment. Resource Popul. Ecol. 32, 187-197.

Toquenaga, Y., Fujii, K., 1991. Contest and scramble competition between two bruchid species (Coleoptra: Bruchidae) II. Larval competition experiment. Resource Popul. Ecol. 33, 129-139.
Tuda, M., 1998. Evolutionary character changes and population responses in an insect host-parasitoid experimental system. Resource Popul. Ecol. 40, 293-299.

Tuda, M., Iwasa, Y., 1998. Evolution of contest competition and its effect on host-parasitoid dynamics. Evol. Ecol. 12, 855-870.

Turchin, P., Taylor, A.D., 1992. Complex dynamics in ecological time series. Ecology 73 (1), 289-305. 